\title{
OPEN Mitochondrial gene signature in the prefrontal cortex for differential susceptibility to chronic stress
}

\author{
Meltem Weger ${ }^{1,5}$, Daniel Alpern ${ }^{2,6}$, Antoine Cherix ${ }^{3,7}$, Sriparna Ghosal $^{1}$, Jocelyn Grosse ${ }^{1}$, \\ Julie Russeil ${ }^{2}$, Rolf Gruetter ${ }^{3}$, E. Ronald de Kloet ${ }^{4}$, Bart Deplancke ${ }^{2,6}$ \& Carmen Sandi $^{1 凶}$
}

Mitochondrial dysfunction was highlighted as a crucial vulnerability factor for the development of depression. However, systemic studies assessing stress-induced changes in mitochondria-associated genes in brain regions relevant to depression symptomatology remain scarce. Here, we performed a genome-wide transcriptomic study to examine mitochondrial gene expression in the prefrontal cortex (PFC) and nucleus accumbens (NAC) of mice exposed to multimodal chronic restraint stress. We identified mitochondria-associated gene pathways as most prominently affected in the PFC and with lesser significance in the NAc. A more detailed mitochondrial gene expression analysis revealed that in particular mitochondrial DNA-encoded subunits of the oxidative phosphorylation complexes were altered in the PFC. The comparison of our data with a reanalyzed transcriptome data set of chronic variable stress mice and major depression disorder subjects showed that the changes in mitochondrial DNA-encoded genes are a feature generalizing to other chronic stress-protocols as well and might have translational relevance. Finally, we provide evidence for changes in mitochondrial outputs in the PFC following chronic stress that are indicative of mitochondrial dysfunction. Collectively, our work reinforces the idea that changes in mitochondrial gene expression are key players in the prefrontal adaptations observed in individuals with high behavioral susceptibility and resilience to chronic stress.

Chronic stress is a major risk factor for the development of psychopathologies, such as depression ${ }^{1-3}$, a severe mental disease with a high prevalence and limited effective treatment possibilities ${ }^{4,5}$. Despite extensive clinical and preclinical research efforts carried out to date (e.g., $\left.{ }^{6-12}\right)$, there is still a lack of understanding about the specific biological changes that link chronic stress with depressive symptoms.

Brain regions, such as the prefrontal cortex (PFC) and the nucleus accumbens (NAc) that are implicated in depression pathogenesis ${ }^{11,13-16}$ show high susceptibility to display structural and functional alterations under exposure to chronic stress ${ }^{12,17-19}$. In the search for potential mechanisms underlying those changes, several studies have reported gene expression changes in the PFC and NAc in both, depressive patients ${ }^{16,20-22}$ and rodent models of chronic stress ${ }^{23-25}$. A very recent comparative study has reported considerable overlap between transcriptional signatures in the PFC and NAc in human major depressive disorder and in three mouse chronic stress models, validating the use of these animal approaches to model depression ${ }^{26}$. Interestingly, only the enrichment of genes involved in mitochondrial function in both PFC and NAc was commonly shared between human depression and every mouse model, whereas for all other shared gene pathways each mouse model captured distinct aspects of major depression abnormalities ${ }^{26}$. While these findings suggest that alterations in brain mitochondrial-related gene expression may be a critical evolutionarily conserved pathway linking chronic stress with depression, this study notably did not follow up on the mitochondrial data ${ }^{26}$.

${ }^{1}$ Laboratory of Behavioral Genetics, Brain Mind Institute, École Polytechnique Fédérale de Lausanne, 1015 Lausanne, Switzerland. '2Laboratory of Systems Biology and Genetics, Institute of Bioengineering, École Polytechnique Fédérale de Lausanne, 1015 Lausanne, Switzerland. ${ }^{3}$ Laboratory for Functional and Metabolic Imaging, École Polytechnique Fédérale de Lausanne, 1015 Lausanne, Switzerland. ${ }^{4}$ Departement of Endocrinology and Metabolic Disease, Leiden University Medical Center, Leiden, The Netherlands. ${ }^{5}$ Institute for Molecular Bioscience, The University of Queensland, St. Lucia, QLD 4072, Australia. ${ }^{6}$ Swiss Institute of Bioinformatics, 1015 Lausanne, Switzerland. ${ }^{7}$ Present address: Nuffield Department of Clinical Neurosciences, John Radcliffe Hospital, University of Oxford, Oxford OX3 9DU, England, UK. ${ }^{\varpi}$ email: carmen.sandi@epfl.ch 
Mitochondria are multifunctional life-sustaining organelles responsive to life stress and increasingly recognized as potential intersection points between psychosocial experiences and biological stress responses ${ }^{27,28}$. For example, stress-induced mitochondrial impairments leading to decreased ATP synthesis and/or excessive production of reactive oxygen species (ROS) may affect behavior by interfering with neuroplasticity and causing neurotoxicity ${ }^{28-30}$. Indeed, mitochondrial dysfunction is emerging as a vulnerability factor for human depression $^{31,32}$ and stress-related psychopathologies ${ }^{28,30}$. However, information regarding the precise stressinduced changes in mitochondria-associated genes in brain regions relevant for depression symptomatology, and how they relate to depression-related behavioral manifestations, is scarce. Only a few studies have investigated alterations in the transcriptional expression of mitochondria-associated genes in the brain of major depression disorder (MDD) subjects ${ }^{33}$, including the $\mathrm{PFC}^{34}$. In rodent models of chronic stress, a recent multi-omics study including genome-wide analyses identified mitochondrial-related alterations in gene expression in the bed nucleus of stria terminalis, an anxiety-related brain region ${ }^{35}$.

Here, we performed a genome-wide transcriptomic study using bulk transcriptomics to assess and define mitochondrial gene expression signatures in the PFC and NAc upon chronic stress. We firstly investigated whether mitochondrial gene sets and pathways would show enriched differential expression in the PFC and NAc between mice submitted to multimodal chronic restraint stress (mCRS) and unstressed control (CTR) mice. As our results revealed prominent chronic stress-induced alterations in mitochondrial pathways, we then performed a detailed analysis to understand to which extent nuclear (nDNA) and mitochondrial DNA (mtDNA)-encoded mitochondria-related genes were affected in these brain regions. In addition, to assess whether the detected mitochondrial gene expression changes are a chronic stress feature that generalizes to other protocols, we reanalyzed a previously published gene expression data set of PFC and NAc from chronic variable stress (CVS) mice ${ }^{20}$. Moreover, to provide translational implications of our findings, we compared our data with differential gene expression in major depression disorder (MDD) subjects ${ }^{20}$. Lastly, we provide evidence that the here defined mitochondrial gene expression signature reflects the mice behavioral profiles and their susceptibility to chronic stress.

\section{Results}

The mCRS protocol leads to changes in physiology and behavior. Mice were randomized into CTR or mCRS groups following matching according to their body weight and anxiety-like behaviors (i.e., percent time in the open arms of the EPM; see experimental schedule in Fig. 1A). The OF and NO tests confirmed that there were no a priori group differences in anxiety, locomotion, and exploration (Fig. S1A,B). During exposure to mCRS, stressed mice showed reduced body weight gain and lower food intake at the beginning of the stress paradigm than CTRs (Fig. 1B). Stress also led to increased basal blood CORT levels, as measured four days after the end of the mCRS protocol (Fig. 1C) and increased adrenal glands' weight (Fig. 1D).

At the behavioral level, and as compared to CTRs, mice exposed to mCRS showed: (1) decreased sociability, as indicated by their reduced time spent in the social interaction zone and increased social avoidance score (Fig. 1E); (2) increased immobility in the FST (Fig. 1F); and (3) unaltered saccharin preference (Fig. 1G). Importantly, the computed integrated $\mathrm{z}$-score indicated that mCRS exposed animals showed a significant alteration when the performance across the different tested behavioral domains was considered (Fig. 1H).

mCRS severely affects the PFC transcriptome and leads to changes in mitochondrial pathways. Next, we performed a hypothesis-free transcriptomic analysis to explore the effects of mCRS on PFC and NAc gene expression (see Table S1 for a list of differentially expressed genes in the PFC and NAc, and Fig. S2A, B for mapping efficiency). The overall number of differentially expressed genes in the PFC following mCRS exposure was higher than the number of differentially expressed genes in the NAc (Fig. 2A-C). The top 20 differentially expressed genes contained genes previously implicated in stress-vulnerability and/or depression (for details, see Fig. S2C,D), indicating that mCRS leads to pathological changes in the PFC and NAc transcriptomes. We then performed a gene set enrichment analysis to identify the most affected pathways in the PFC and NAc (Table S2) upon mCRS. The top 10 enriched pathways in the PFC, interestingly, included gene pathways implicated in mitochondrial energy synthesis, such as "oxidative phosphorylation", "aerobic electron transport chain", and "mitochondrial electron transport" (Fig. 2D). In line with these findings, translation (e.g., "ribosome" and "cytoplasmic translation") as a cellular energy-depending process was also affected in the PFC (Fig. 2D). Gene pathways implicated in mitochondrial energy synthesis were also detected in the NAc (e.g., "oxidative phosphorylation", "energy coupled proton transport"; Fig. 2E), although with a lower significance than in the PFC. These observations highlight profound transcriptional imbalances in mitochondrial gene expression upon mCRS particularly in the PFC.

Gene expression profiles of mitochondria-associated genes following mCRS. Our former analyses pointed to changes in mitochondrial pathways upon mCRS in the PFC, and to some extent in the NAc. We next focused on the mCRS-induced changes in mitochondria-associated genes to decipher the contribution of nDNA and mtDNA encoded genes. mCRS led to an up-regulation of mitochondria-associated genes encoded by both nDNA and mtDNA encoded genes in the PFC (Fig. 3A). A deregulation of mitochondria associated genes was not detected in the NAc (Fig. 3A). Specifically, all of the detected mtDNA encoded genes of the oxidative phosphorylation (OXPHOS) complexes I, III and IV (i.e., $m t-N d 1, m t-N d 2, m t-N d 4, m t-N d 5, m t-N d 6, m t-C y t b$, and $m t$-Co1) were up-regulated in the PFC of mCRS mice (Fig. 3B,C). Note that the other six mtDNA encoded genes of the OXPHOS complexes (i.e., $m t-N D 3, m t-N D 4 L, m t-C o 2, m t-C o 3, m t-A t p 6$, and $m t$-Atp8) were too low expressed to be reliably detected in our study. We verified the expression changes of selected mtDNA encoded genes by qRT-PCR and confirmed upregulation of the complex I genes $m t-N d 1$ and $m t-N d-3$ (Fig. S3). To obtain 
A

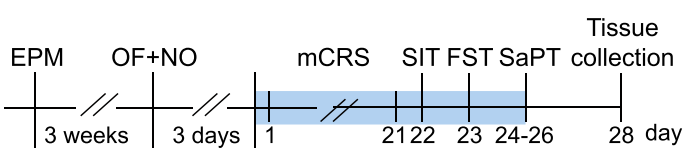

B

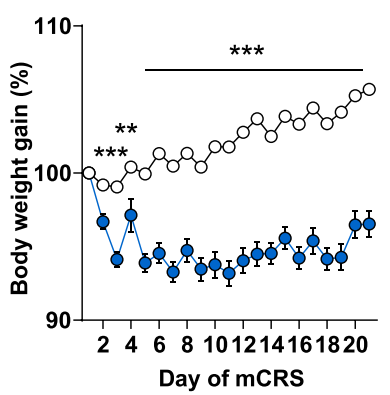

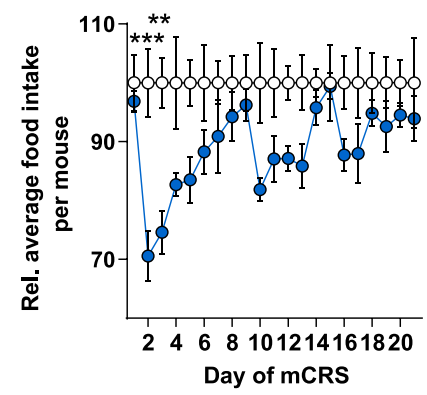

C

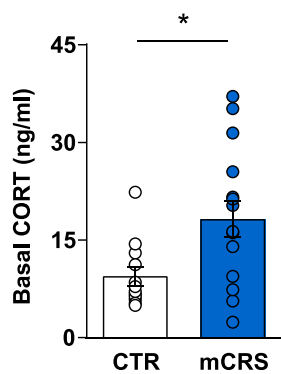

$\mathbf{F}$

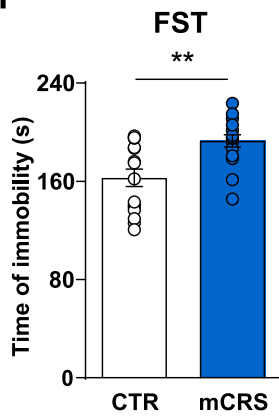

D
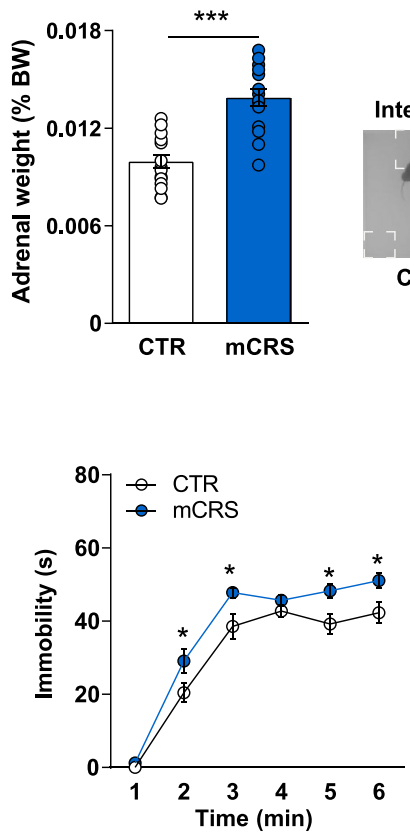

E

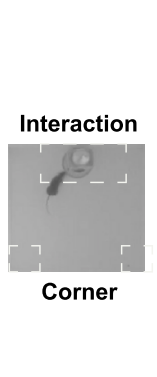

G

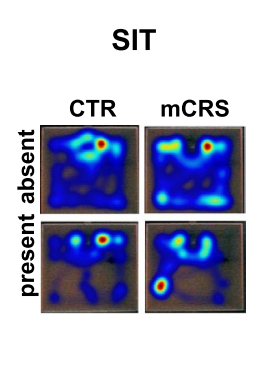

SaPT

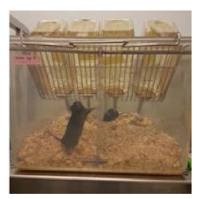

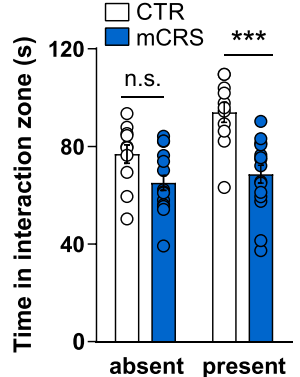

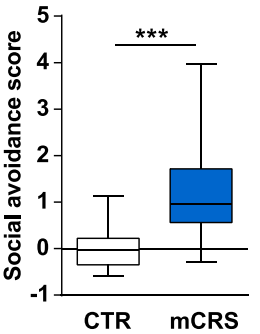

H
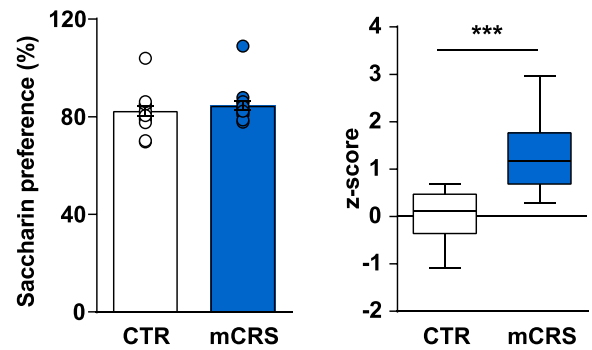

Figure 1. Effects of chronic stress in physiology and behavior. (A) Experimental timeline: Mice were randomized in two groups according to body weight and performance in the elevated plus maze (EPM) and open field and novel object (OF + NO) tests: unstressed controls (CTR) and mice submitted to multimodal chronic restrain stress (mCRS) $\mathrm{N}=16$ /group. From mCRS day 21, they were tested in the social Interaction (SIT), forced swim (FST), and saccharine preference (SaPT) tests. Tissue was collected on day 28, 4 days after the last stress session. (B) Changes in body weight (stress effect: $\mathrm{F}_{1,30}=94.67, p<0.0001$; interaction: $\mathrm{F}_{20,600}=35.42, p<0.0001$; repeated measures two-way Anova, Sidak's multiple comparison test; $\mathrm{n}=16 /$ group) and food intake weight (stress effect: $\mathrm{F}_{1,6}=4.64, p=0.0747$; interaction: $\mathrm{F}_{20,120}=3.841, p<0.0001$; repeated measures two-way Anova, Sidak's multiple comparison test; $\mathrm{n}=16$ /group). (C) Post-mCRS basal corticosterone (CORT) levels on day of sacrifice (Mann-Whitney U-test, two-tailed; $n=12$ CTR, $n=15$ mCRS). (D) Post-mCRS adrenal glands weight (Unpaired t-test, two-tailed; $\mathrm{n}=15$ CTR, $\mathrm{n}=16 \mathrm{mCRS}$ ). (E) SIT: top-down view of the arena and representative heat maps when a social target is absent or present. Barplots indicate the time in the interaction zone (ST presence: $\mathrm{F}_{1,25}=7.88, p=0.0096$; stress effect: $\mathrm{F}_{1,25}=25.16, p<0.0001$; interaction: $\mathrm{F}_{1,25}=3.352$, $p=0.0791$; repeated measures two-way Anova, Sidak's multiple comparison test; $\mathrm{n}=11 \mathrm{CTR}, \mathrm{n}=16 \mathrm{mCRS}$ ) and the social avoidance score (Unpaired t-test, two-tailed; $n=11$ CTR, $n=16 \mathrm{mCRS}$ ). (F) FST: Immobility time total (Unpaired t-test, two-tailed; $\mathrm{n}=16$ /group) and per min (stress effect: $\mathrm{F}_{1,30}=15.67, p=0.0004$; interaction: $\mathrm{F}_{5,150}=1.553, p<0.1769$; repeated measures two-way Anova, Sidak's multiple comparison test; $\mathrm{n}=16 /$ group). (G) SaPT: Saccharin preference over 3 days (Unpaired t-test, two-tailed; $\mathrm{n}=16 /$ group). (H) Integrated behavioral $\mathrm{z}$-score (computed from $\mathrm{z}$-scores of SIT, FST, and SaPT; Unpaired t-test, two-tailed; $\mathrm{n}=11 \mathrm{CTR}, \mathrm{n}=16 \mathrm{mCRS}$ ). Data are displayed as mean \pm SEM. ${ }^{\star}, p<0.05,{ }^{* *}, p<0.01,{ }^{* * *}, p<0.001$.

information about the expression of complex $\mathrm{V}$ genes, we analyzed $m t$-Atp6 expression and detected its upregulation in the PFC of stressed mice as well (Fig. S3).

To evaluate whether the here detected mitochondrial gene expression changes are a chronic stress feature that generalizes to other chronic stress protocols, we also tested and showed that the up-regulation of both nDNA and mtDNA encoded genes is not solely specific to mCRS but can be also detected following CVS exposure (Fig. 3A, Fig. S4). 
A

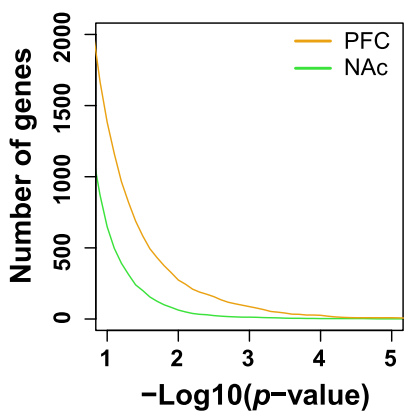

B

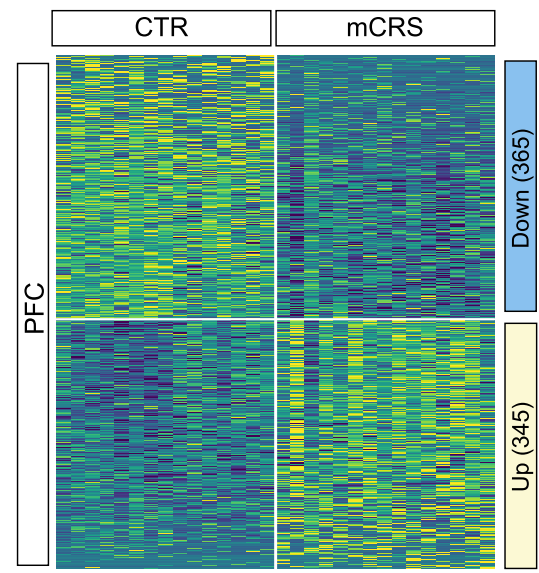

C

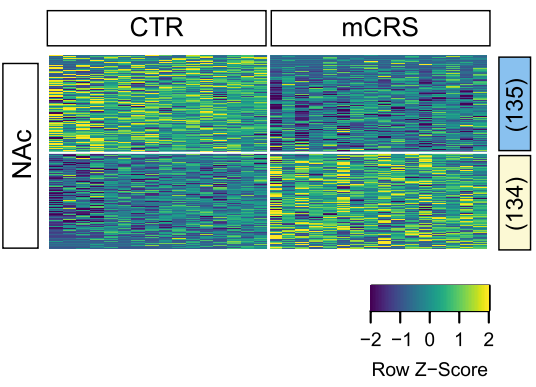

D

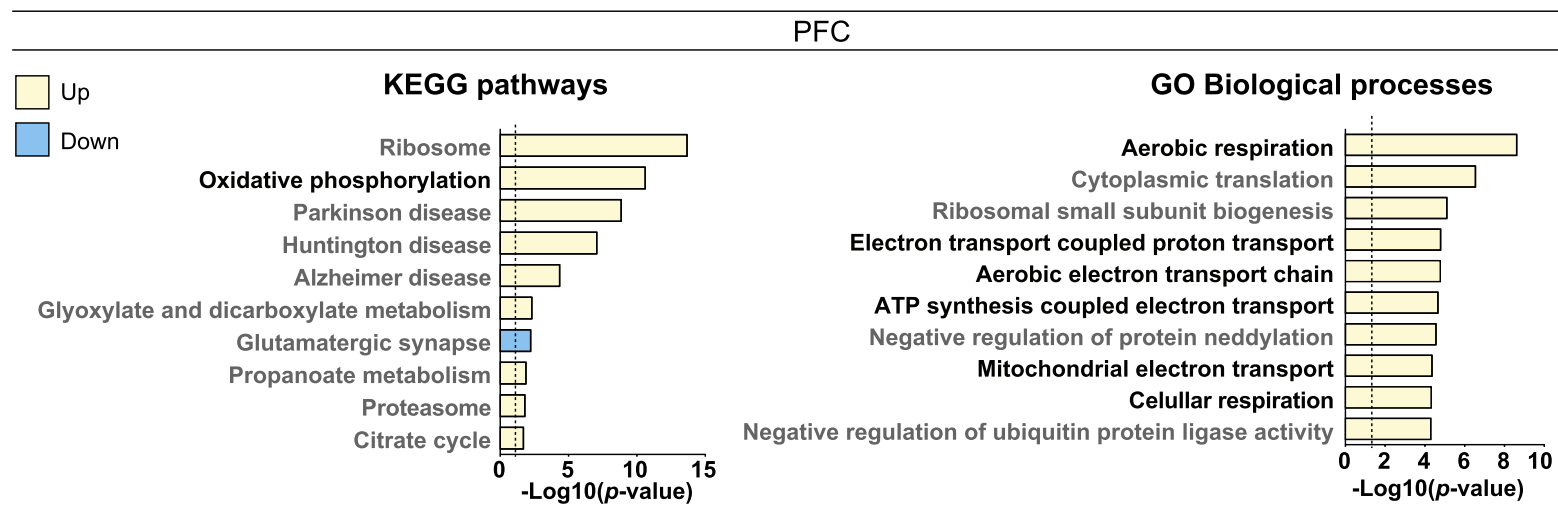

E

\section{NAc}

\section{KEGG pathways}

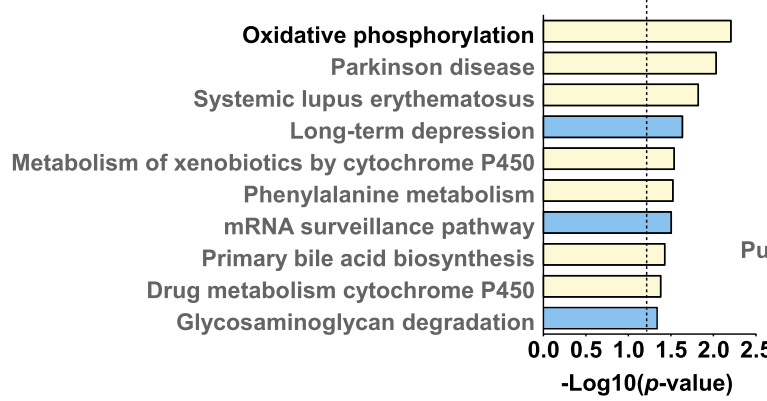

\section{GO Biological processes}

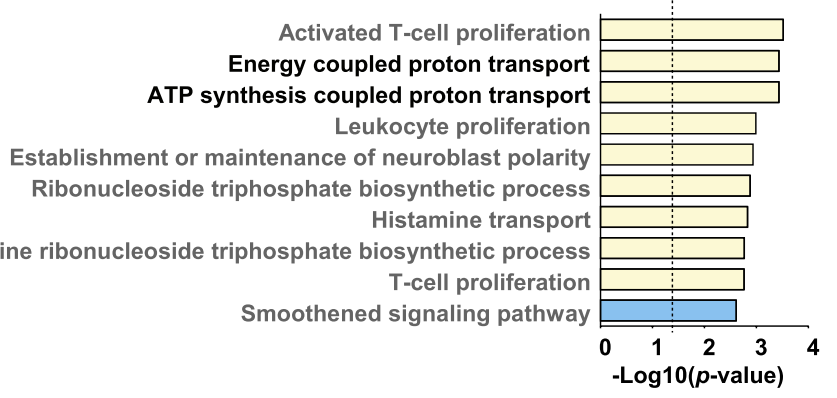

Figure 2. Transcriptional gene expression changes in the PFC and NAc of mice following mCRS. (A) Number of differentially expressed genes (CTR vs. mCRS) in the in the PFC ( $n=15$ /group) and in the NAc ( $n=16$ / group) from mCRS mice and unstressed CTRs in function of the $p$ value. (B) Heatmap of normalized mRNA expression levels of genes in the PFC and (C) in the NAc. Yellow, high expression; blue, low expression. (D) Gene set enrichment analyses of differentially expressed genes in the PFC and (E) in the NAc. Mitochondrial pathways are highlighted in black font. Yellow bars are up-regulated pathways and blue bars are down-regulated pathways.

Furthermore, in a translational effort, we next tested whether the detected mitochondrial gene expression changes in chronically stressed mice are also present under conditions of MDD. For this end, we reanalyzed the data of post-mortem brain tissue from MDD patients and matched control subjects previously published by Labonte et al. ${ }^{20}$. For our comparison, we used the data from vmPFC (henceforth termed PFC) and NAc. Interestingly, there was a high overlap in mitochondria-associated gene expression between the data in mice and MDD subjects for mtDNA-encoded genes in the PFC (Fig. 3A,B). Therefore, altogether these analyses point to mtDNA encoded gene expression changes as particularly susceptible to be altered in the context of chronic stress and depression. 
A

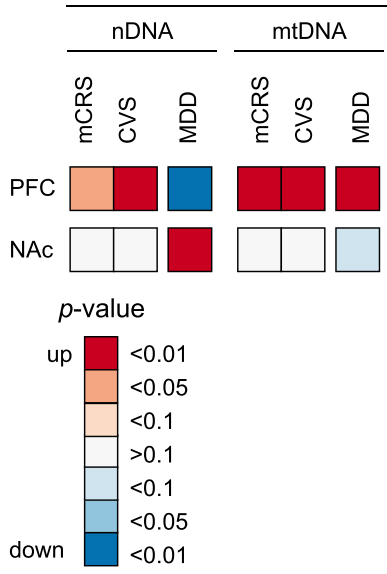

B

PFC

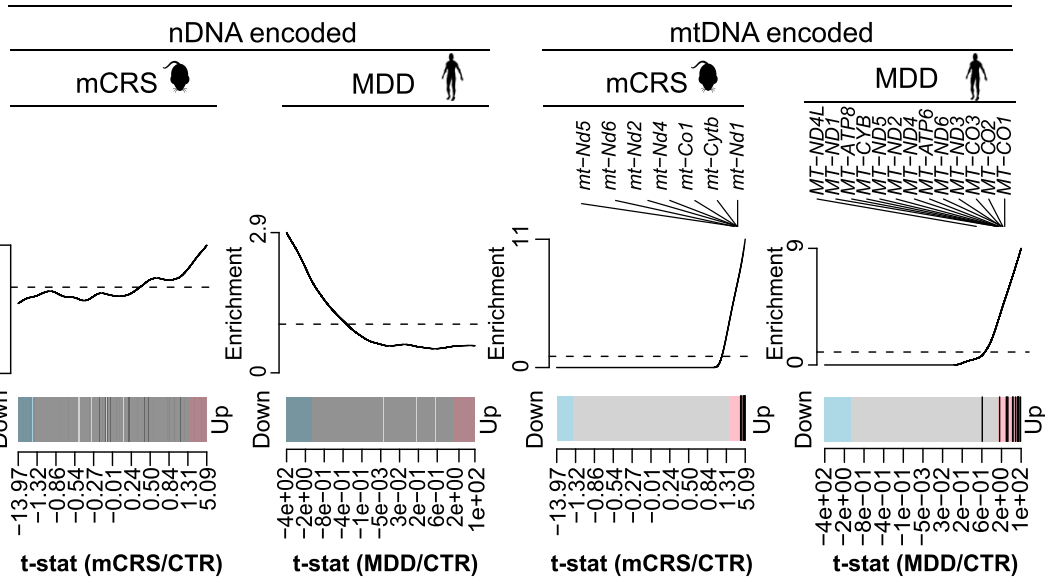

C

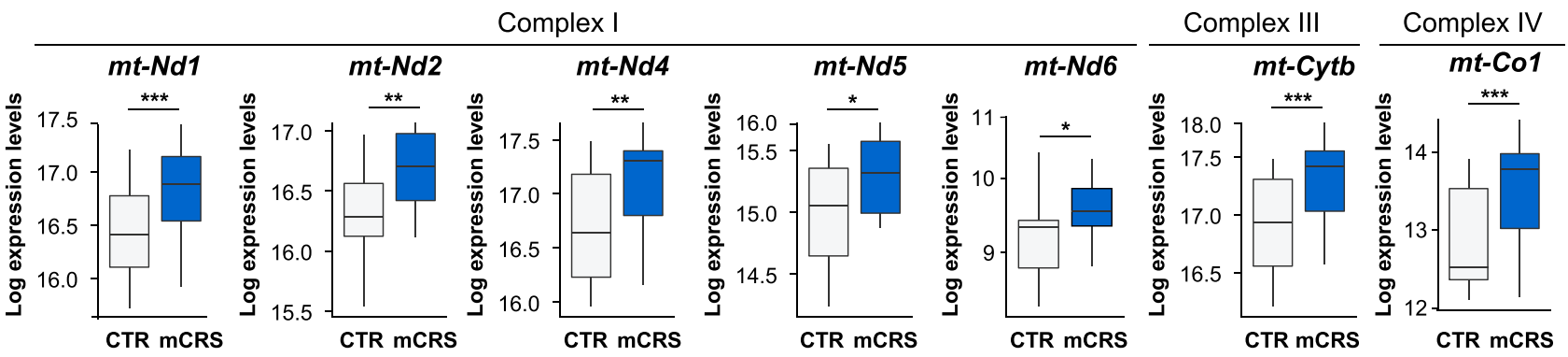

Figure 3. Mitochondria-related gene expression in chronically stressed mice and major depression subjects. (A) Enrichment analyses of mitochondria-associated genes encoded by nuclear DNA (nDNA) or mitochondrial DNA (mtDNA) in mCRS and unstressed CTR mice ( $\mathrm{n}=15$ /group, PFC; $\mathrm{n}=16$ /group, NAc), chronic variable stress (CVS) mice (PFC: $\mathrm{n}=19$ /group, NAc: $\mathrm{n}=20$ /group) and post-mortem tissue of major depression disorder (MDD; $\mathrm{n}=16$, ventromedial (vm) PFC; $\mathrm{n}=16, \mathrm{NAc}$ ) and healthy (CTR; $\mathrm{n}=15$, vmPFC; $\mathrm{n}=17, \mathrm{NAc}$ ) subjects. Red, enrichment for up-regulation; blue, enrichment for down-regulation. Data of CVS mice and MDD subjects were reanalyzed from a previously published transcriptome dataset ${ }^{20}$. (B) Barcode plots for mitochondriaassociated genes encoded by nDNA or mtDNA DNA in the PFC of mice exposed to mCRS and of MDD subjects. (C) Gene expression of mtDNA encoded genes in the PFC of mice ( $p$ values from generalized linear model). Data are displayed as mean \pm SEM. ${ }^{\star}, p<0.05,{ }^{* *}, p<0.01,{ }^{* * *}, p<0.001$.

Chronic stress leads to changes in mitochondrial function and metabolism. The transcriptional changes in mitochondria associated genes indicated the presence of mitochondrial dysfunctions in mCRS mice. Thus, we assessed whether mCRS impacts mitochondrial function in the PFC and the NAc by addressing mitochondrial respiration capacity ex vivo. Our results showed a reduction in mitochondrial respiration capacity in mCRS mice in the PFC (Fig. 4A) but no changes in the NAc (Fig. 4B). To gain more insights on the impact of mCRS on brain metabolism, we performed ${ }^{1} \mathrm{H}$-magnetic resonance spectroscopy $\left({ }^{1} \mathrm{H}\right.$-MRS; Fig. S5) and evaluated energy-related (i.e., creatine, phospho-creatine, glucose) metabolites, including glycolytic (i.e., alanine, lactate) ones ${ }^{36}$. Levels of glucose (Glc), the brain's primary energy source, were decreased in the PFC of mCRS mice (Fig. 4C). No mCRS-induced changes in metabolites were detected in the NAc (Fig. 4D).

Next, we tested whether mCRS induces the mitochondrial unfolded stress-response (UPR $\left.{ }^{\mathrm{mt}}\right)$ which is initiated by the transcription factors ATF-5, ATF-4 and CHOP/DDIT3 ${ }^{37-39}$ upon mitochondrial dysfunctions to maintain homeostasis ${ }^{40}$. To predict in silico for potential transcription factor binding sites and thus activity of ATF-5, ATF-4 and CHOP/DDIT3 ("predicted motif activity") in the promoters of differentially expressed genes in the PFC and NAc upon mCRS, we scanned $\pm 500 \mathrm{bp}$ around the annotated transcription start site of a gene. Interestingly, our analysis revealed that the predicted ATF-4 and CHOP/DDIT3 motif activity is higher in the PFC of mCRS mice compared to CTR animals (Fig. 4E). In contrast, no increased predicted motif activity of any of the tested transcription factors was detected in the NAc of mCRS mice (Fig. 4F), indicating that the UPR ${ }^{\mathrm{mt}}$ via the ATF4 pathway due to mitochondrial dysfunctions is active in the PFC of mice following mCRS.

mtDNA encoded gene expression in the PFC correlates with mice behavioral profiles. To gather more information about whether the expression of mitochondria-associated genes in the PFC of mCRS mice can be related to mice behavioral profiles, we applied a model selection analysis of gene expression that considers the individual differences in stress and behavioral responses of the tested mice. Specifically, this method allows to extract genes either better correlating with 1) the treatment itself (i.e., mCRS) or with 2) the 
PFC

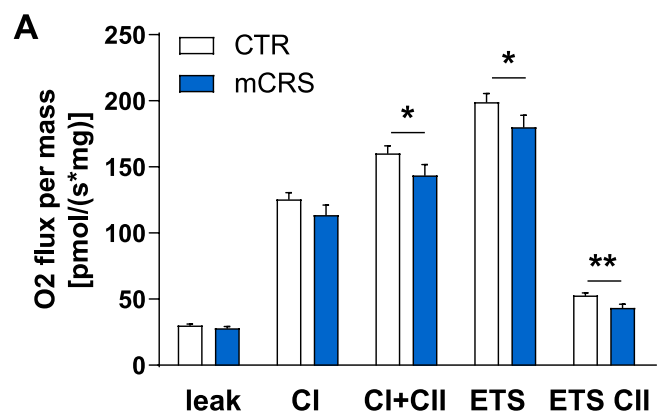

C

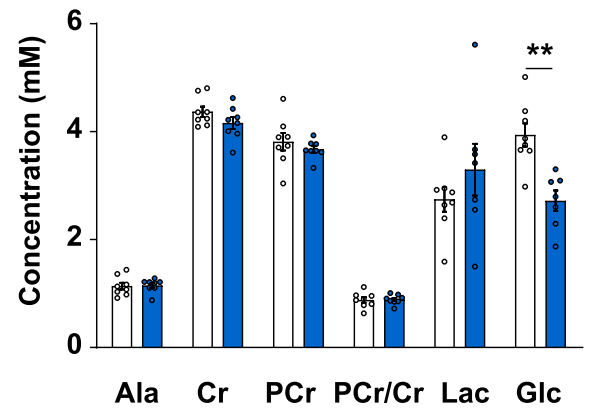

NAc

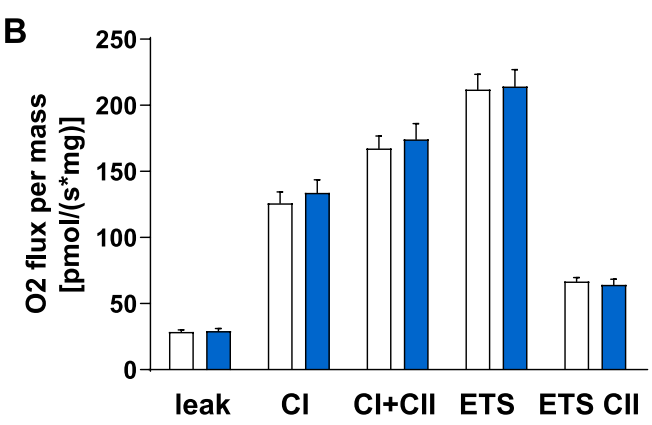

D

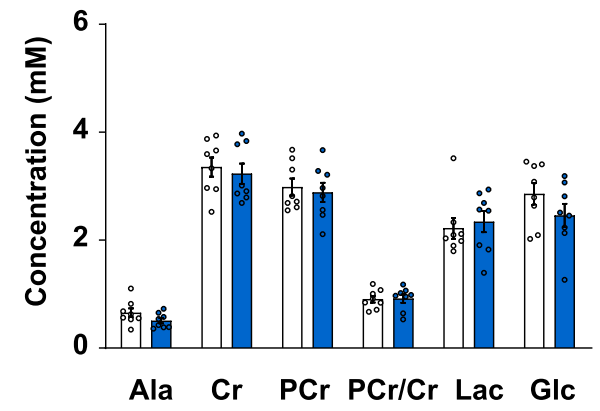

E

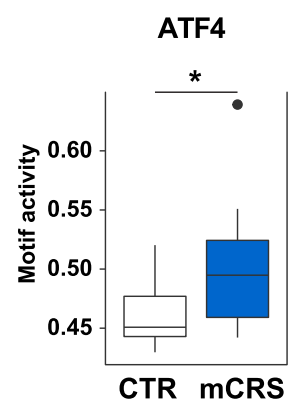

CHOP/DDIT3

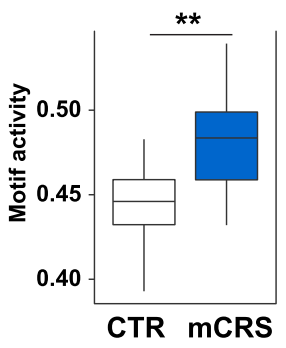

ATF5

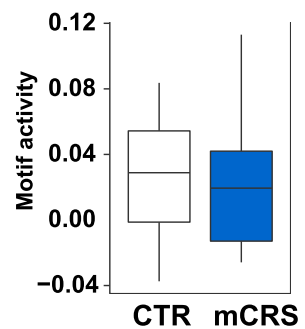

ATF4

CHOP/DDIT3

ATF5

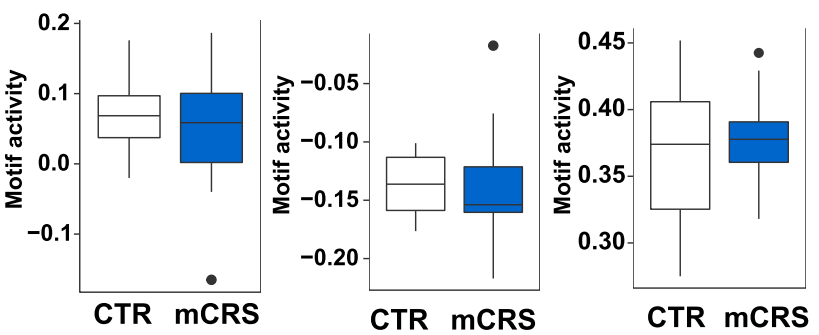

Figure 4. Effects of chronic stress on mitochondrial respiration and metabolites, and in silico transcription factor activity analysis in the PFC and NAc. (A) Ex vivo analysis of mitochondrial respiration in the PFC ( $p$ values from linear mixed models; $n=16$ CTR, $n=15 \mathrm{mCRS}$ ). (B) Ex vivo analysis of mitochondrial respiration in the NAc ( $p$ values from linear mixed models; $n=16$ /group). (C) In vivo ${ }^{1} \mathrm{H}-\mathrm{MRS}$ measurements of energy metabolites in the PFC and (D) in the NAc ( $p$ values from linear models; $n=8 /$ group). Abbreviations: Ala, alanine; Cr, creatine; PCr, phosphocreatine; Lac, lactate; Glc, glucose. (E) In silico analysis of BRB-seq data to determine potential transcription factor binding sites of ATF-5, ATF-4 and CHOP/DDIT3 in the promoters of differentially expressed genes in the PFC upon mCRS (Unpaired t-test, two-tailed; $n=15$ /group) and $(\mathbf{F})$ in the NAc upon mCRS (Unpaired t-test, two-tailed; $\mathrm{n}=16$ /group). Data are displayed as mean $\pm \mathrm{SEM} .{ }^{*}, p<0.05,{ }^{* *}$, $p<0.01$.

consequences of the treatment on behavior (i.e., z-score). In our analysis, the glucocorticoid-dependent mTOR regulator Ddit $4^{41}$ would be an example for genes of group 1 and the mitochondrial gene $N d u f v 2$ would represent genes of group 2 (Fig. 5A, Table S1).

A gene set enrichment analysis of gene pathways better correlating with the behavioral profiles of mCRS mice led to the observation that "oxidative phosphorylation" is one of the pathways that is better explained by individual behavioral changes ( $\mathrm{z}$-score) than simply the absence or presence of mCRS (Fig. 5B, Table S2). A more detailed analysis of the OXPHOS complex genes showed that genes of complex I, but not of the other complexes, correlate better with the integrated behavioral z-score than with mCRS (Fig. 5C). In fact, in our study all detected mtDNA encoded genes correlated better with the $z$-score than with mCRS (Fig. 5D,E). In addition, we reevaluated our data by separating mCRS animals according to their susceptibility to stress [i.e., high- (HS) versus low-susceptible (LS), in accordance with $\left.{ }^{42}\right]$. This approach showed that mCRS causes increased expression of mtDNA encoded genes only in HS but not in LS animals (Fig. 5F). 
A

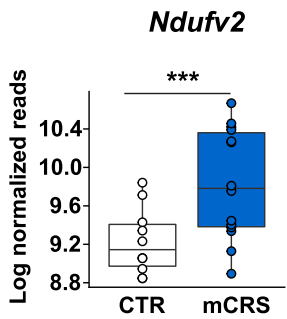

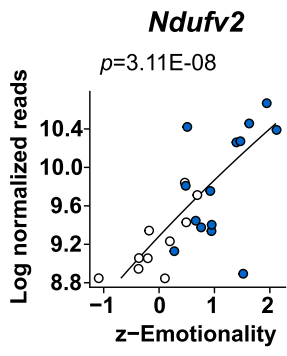

C
B

E

mCRS

Z-score

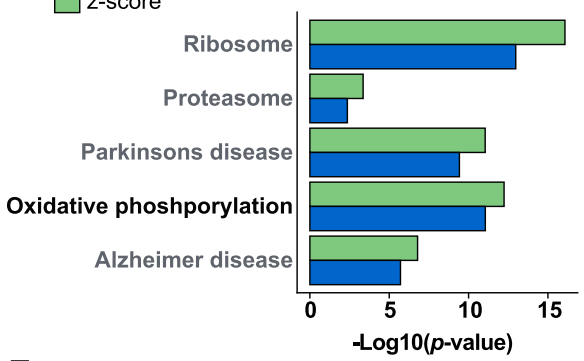

mCRS

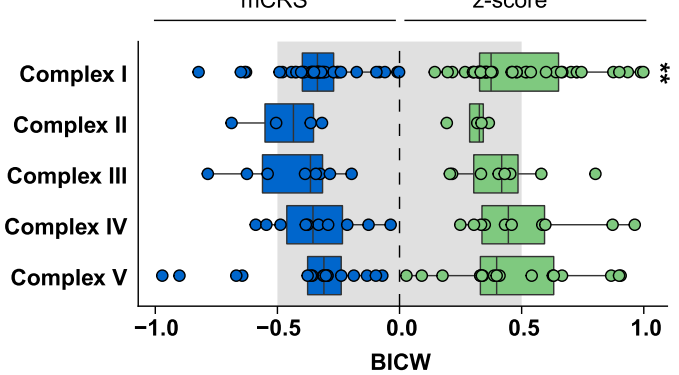

Ddit4
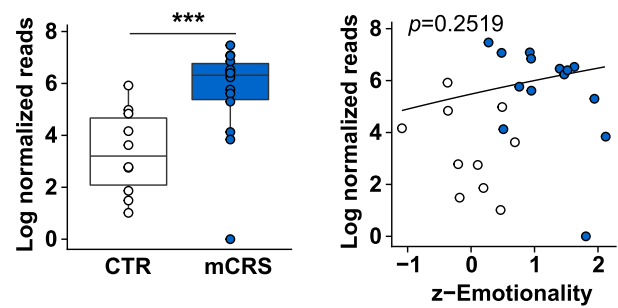

D
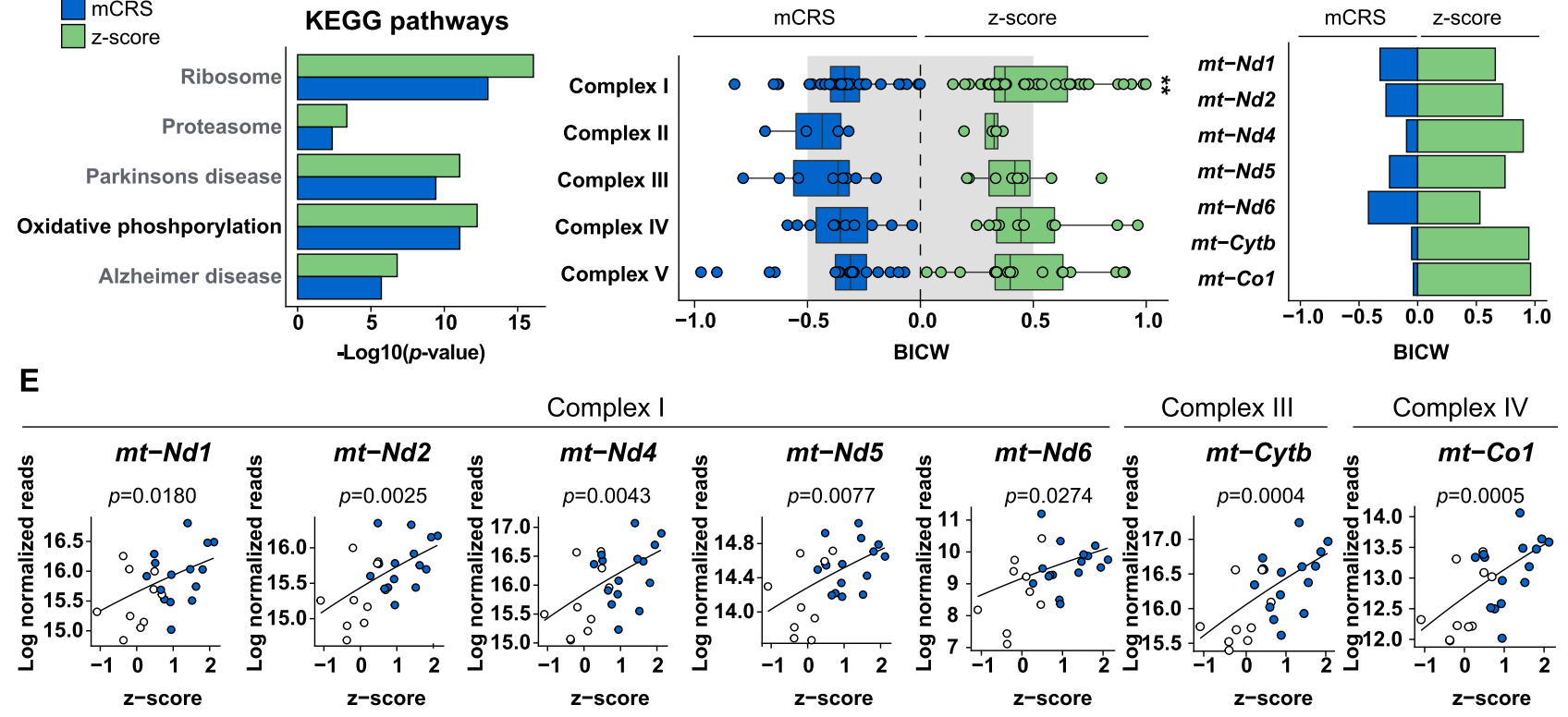

F $\quad m t-N d 1$
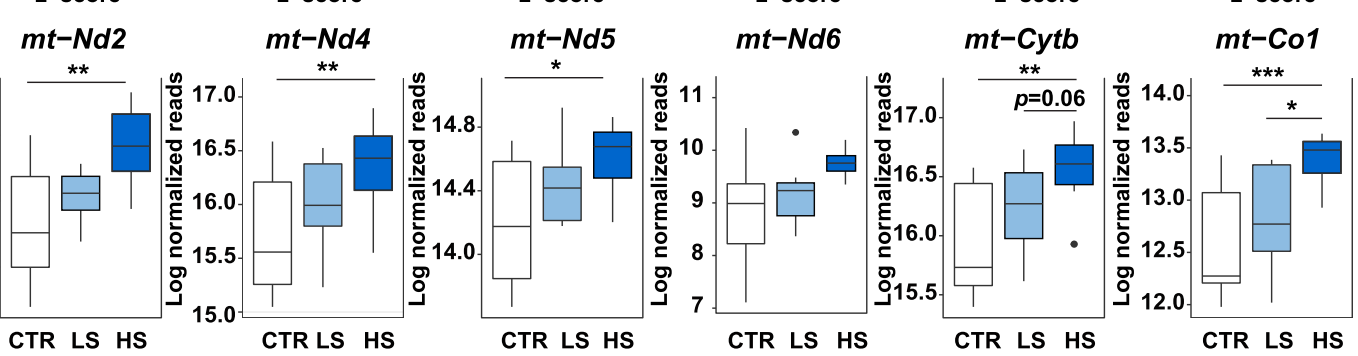

Figure 5. Model selection and stress susceptibility analysis of mCRS gene expression for CTR and high- and low-susceptible mCRS mice. (A) The gene expression profile of $N d u f v 2$ is better captured by a generalized linear model considering individual behavioral profiles (z-score) than a model that only considers the absence or presence of mCRS. On the other hand, Ddit4 expression is better explained by the mCRS model than by the z-score model. (B) Gene pathways better described by the z-score than by mCRS. Oxidative phosphorylation is highlighted in black font. (C) Results of the model selection approach for genes associated with the indicated OXPHOS complexes. Genes associated with OXPHOS complex I better correlate with the integrated behavioral z-score than with mCRS. BICW, Bayesian information criterion weight. (D) Results of the model selection approach for detected mtDNA encoded genes. Gene expression of all detected mtDNA encoded genes are better explained by a model that relies on the integrated behavioral z-score rather than presence or absence of mCRS.

(E) Correlational analyses between normalized mtDNA-encoded genes and integrated behavioral z-score.

(F) Levels of mtDNA encoded genes from different OXPHOS complexes in CTR animals and mCRS mice grouped into high- (HS) or low-susceptibility (LS) to stress according to their integrated $\mathrm{z}$-score ( $p$ values from generalized linear model). $\mathrm{n}=11 \mathrm{CTR}, \mathrm{n}=15$ mCRS. Data are displayed as mean \pm SEM. ${ }^{*}, p<0.05,{ }^{* *}, p<0.01$, $* * *, p<0.001$.

\section{Discussion}

Mitochondrial dysfunction has been postulated as a key etiopathology factor for the development of chronic stress-induced psychopathological alterations, including depression ${ }^{28,30}$. Neural adaptations to chronic stress 
are energetically costly and mitochondria are ideally positioned to contribute to them by providing energy and stress signaling molecules ${ }^{28,30}$. However, systematic studies describing the precise alterations in mitochondriaassociated genes in the brain following exposure to chronic stress remain scarce. In this study, using a genomewide hypothesis-free transcriptomic approach, we identified mitochondria-associated gene pathways among the categories showing the most prominent responses in the PFC and to a lesser extent in the NAc following chronic stress exposure in mice. Most importantly, we revealed that mtDNA genes coding for different subunits of the OXPHOS complexes in the PFC as particularly affected by stress, and showed that their upregulation is particularly observed in stress-vulnerable mice.

First, we confirmed that our multimodal chronic restraint stress protocol leads to reduced sociability and increased passive coping responses under adversity, two behavioral alterations also typically observed in depressive individuals ${ }^{43-45}$. Then, the computation of the number of genome-wide stress-responsive genes, including mitochondria-associated ones, pointed at a higher reactivity of the PFC to stress than the NAc. This observation is in line with other reports showing that, at the transcriptional level, the PFC is more severely affected than the NAc by chronic stress or major depression ${ }^{20,24,46}$. Rodent studies have reported opposite effects of chronic stress in neuronal structure in these two brain regions, frequently indicating decreases in spine density and dendritic complexity in the PFC ${ }^{47-50}$ while increase in the NAc ${ }^{49,51}$ (but se ${ }^{52}$ ). However, a note of caution should be added here, not only because the evidence is mixed as indicated above, but also because different cell types are involved in the referred studies. While data from the NAc has been obtained from the analysis of GABAergic projection neurons (i.e., medium spiny neurons), the atrophy described in the PFC refers to excitatory pyramidal cells. In fact, opposite results (i.e., dendritic hypertrophy) have been found in the medial PFC when analyzing the effects of chronic stress in a subpopulation of interneurons ${ }^{53}$. However, the relatively lower susceptibility of the NAc to show transcriptomic changes following chronic stress, including mitochondria-associated ones, contrasts with the highest vulnerability shown by the NAc than the PFC to display changes in neurometabolic markers following stress $^{18}$. A possible reason for this discrepancy may be the different stress protocols used (i.e., 10-days chronic social defeat ${ }^{18}$ vs. the here applied 3-week mCRS). Note, however, that a 3-way chronic restraint protocol results as well in major metabolic changes in the NAc in stress-vulnerable mice ${ }^{54}$. In the future, it will be important to integrate transcriptomic and metabolic analyses in these two brain regions, ideally at the cell-type level, to further our understanding of stress-induced metabolic regulation at the transcriptional level.

Mitochondria are multifunctional life-sustaining organelles that have as a major function to perform OXPHOS, the metabolic pathway in which the electron transport chain, through its complexes I-IV, exchanges protons and electrons to eventually produce adenosine triphosphate (ATP) by complex V. Subunits of the mitochondrial complexes of OXPHOS are encoded not only by nDNA but also by mtDNA ${ }^{28}$. Our mitochondriafocused analysis showed that mCRS mice exhibited an up-regulated expression of mtDNA encoded genes of virtually all OXPHOS complexes, except for complex II. We furthermore showed that mtDNA encoded genes are also up-regulated in the PFC of mice subjected to another chronic stress paradigm ${ }^{20}$. These results align well with proteomic data indicating chronic stress-induced changes in the levels of proteins involved in mitochondrial transport and OXPHOS in the PFC ${ }^{55}$. Likewise, chronic stress was also shown to lead to up-regulated expression of the mtDNA-encoded complex I gene mt-ND6 in the rat hippocampus ${ }^{56}$. Altogether, these findings, strongly supported the notion that an upregulated expression of mtDNA encoded genes is a common response to chronic stress. Importantly, mouse gene deletion approaches involving genes of the mitochondrial OXPHOS complexes were shown to lead to alterations in physiological stress responses ${ }^{27,57}$.

Importantly, we found stress-induced increases in the expression of genes coding for subunits of different complexes of the OXPHOS machinery in the PFC along with decreased levels of the energy metabolite glucose and reduced maximal capacity of mitochondrial respiration as evaluated ex vivo. Diminished mitochondrial respiration in the PFC was also reported for other chronic stress models ${ }^{58}$ and in fibroblasts from major depressive disorder patients ${ }^{59}$. Glucose levels are also typically found decreased in the brain of depressed patients ${ }^{60-62}$ and following chronic stress in rodents ${ }^{63}$, in association with a reduction in glucose transporters expression ${ }^{63}$. Given that glucose is the main source of energy for the mammalian brain ${ }^{64}$, through a key contribution to OXPHOS processes ${ }^{65}$, the observed increases in genes coding for OXPHOS complexes subunits may be an allostatic response to compensate for reduced fuel ${ }^{28,66,67}$. However, given that our neurobiological endpoints were only measured after chronic stress exposure, we cannot discard that they are not a reaction to stress-induced enhancements in mitochondrial function potentially observed at earlier time points ${ }^{56,68}$, particularly in the PFC $^{69}$. Eventually, the reported compensatory changes could represent responses to the "allostatic load" induced by chronic stress ${ }^{70}$. Such a view would reconcile the reductions in dendrite complexity and spine density typically observed following chronic stress in the $\mathrm{PFC}^{47,48}$ and the increased neuronal vulnerability triggered by elevated rate of mitochondrial OXPHOS ${ }^{71,72}$. Increased rate of mitochondrial OXPHOS would lead to enhanced production of ROS making neurons more vulnerable and less capable of sustaining complex axonal arborization ${ }^{71,73}$. This view is further reinforced by the fact that the increased expression of mtDNA encoded OXPHOS genes upon chronic stress relates with the behavioral profiles of the mice and was particularly observed in high-susceptible animals. Altogether, our data point to that mCRS leads to profound changes in mitochondrial outputs in the PFC that are indicative for mitochondrial dysfunction in this brain region.

Our findings of high transcriptional susceptibility of mitochondrial pathways to stress align well with the recent observation of similar marked dysregulations across three mouse models of chronic stress and in human MDD both in the PFC and in the NAc ${ }^{26}$. Here, we go beyond those observations and provide translational evidence for similar upregulation of mtDNA encoded OXPHOS genes in the PFC of subjects with major depression disorder, a new finding we made by reanalyzing a previously published transcriptome data set ${ }^{20}$. Altogether, these findings reinforce the idea that changes in mitochondrial gene expression are key players in the prefrontal adaptations observed in individuals with high behavioral susceptibility to chronic stress. 
In the future, it will be important to perform similar mitochondrial analyses as the ones included in the current study to investigate chronic stress effects in other brain regions critically involved in the brain response to chronic stress and MDD, such as the orbitofrontal cortex (OFC), hippocampus and amygdala ${ }^{74,75}$. These studies will be particularly relevant to understand the link between mitochondria and stress-induced changes in neuronal structure as, in contrast to the hypotrophy induced by stress in the medial $\mathrm{PFC}^{47-50}$ or in the hippocampus ${ }^{76,77}$, the basolateral amygdala ${ }^{78}$ (but see ${ }^{79}$ ) and the OFC show dendritic hypotrophy ${ }^{80}$ following similar stress protocols. In addition, future functional studies will be needed to elucidate the underlying molecular mechanisms of how the herein defined mitochondrial gene expression changes can act as a vulnerability factor for the development and/or pathology of stress-induced depression. Some evidence from the literature points to glucocorticoids via the glucocorticoid receptor (GR) as potential mediators. Previous studies have shown that both mitochondrial function $^{81}$ and glucocorticoid actions ${ }^{82,83}$ in the NAc is important for the establishment of social dominance in rats, a considered vulnerability factor for depression ${ }^{84}$. Moreover, the GR implicated in the mesocorticolimbic dopamine system with chronic stress vulnerability ${ }^{85,86}$, and showed that the GR has the capability to translocate into brain mitochondria ${ }^{68,87,88}$ and to bind on the mitochondrial genome ${ }^{56,89}$ in a glucocorticoid-dependent manner ${ }^{56}$. It will also be important to test whether mitochondrial-targeted interventions known to ameliorate stress and anxiety responses ${ }^{54,90-92}$ would reverse both the behavioral and mtDNA transcriptional changes observed in stress-vulnerable mice.

In summary, we here defined the mitochondrial gene signatures upon chronic stress in the PFC and NAc, two key brain regions implicated with stress-vulnerability and depression. Our data point to a higher susceptibility of the PFC to chronic stress on a transcriptional level than of the NAc. Moreover, our studies indicate that chronic stress induces a conserved response in mtDNA encoded gene expression in the PFC. Future studies will elucidate how these mitochondrial gene signatures might mediate differential susceptibility to chronic stress.

\section{Material and methods}

Animals. Experimental male 6-week old C57Bl6/J mice and retired old-breeder CD1 mice serving as social targets for the SIT test were purchased from Charles River Laboratories. Experimental animals were housed four per cage (except for the CD1 mice that were due to the experimental design under single-housed conditions) and were allowed to acclimate to the vivarium for at least one week. Animals were handled for at least 1 min per day for three subsequent days and then were weighted upon arrival and then weekly to ensure good health. Animals were kept under standard housing conditions on corn litter in a temperature- $\left(23 \pm 1^{\circ} \mathrm{C}\right)$ and humidity- $(40 \%)$ controlled animal room with a $12 \mathrm{~h}$ light/dark cycle $(7 \mathrm{am}-7 \mathrm{pm})$ with ad libitum access to food and water. All animal procedures were conducted in accordance with the regulations and approved by the Cantonal Ethics Committee of the Canton Vaud, Switzerland.

Behavioral and physiological characterization. Mice (7 weeks old) were randomized in two equivalent groups according to their body weight and performance in the elevated plus maze (EPM). They were then exposed at the age of 10 weeks to open field and novel object $(\mathrm{OF}+\mathrm{NO})$ tests to verify the effectiveness of the randomization. Starting at an age of 11 weeks one group of mice was then exposed to chronic multimodal chronic restraint stress (mCRS; $\mathrm{n}=16$ ), the other was kept as unstressed controls (CTR; $\mathrm{n}=16$ ). Body weight and food intake was measured daily before stress exposure. Mice (14 weeks old) were then tested in the social interaction test (SIT), the forced swim test (FST), and the saccharin preference test (SaPT). Trunk blood was collected during sacrifice ( 15 weeks old) for basal plasma corticosterone (CORT) measurements and the adrenal glands were dissected to determine adrenal glands' weight.

EPM. The EPM was performed under baseline conditions to randomize animals into experimental groups according to their anxiety levels, a vulnerability factor for depression ${ }^{93}$. The apparatus consisted of black PVC with a white floor with two open arms and two closed arms $(30 \times 5 \mathrm{~cm})$ placed $65 \mathrm{~cm}$ above the floor. Light conditions during the testing were $12 \mathrm{~lx}$ in the open arms and 3-4 lx in the closed arms. Experimental animals were placed into the center of the EPM and exploratory behavior were analyzed for 5 min with a video-tracking software (Ethovision 11.0 XT, Noldus, Information Technology). The time an experimental animal spent in the open arms, center, and closed arms was measured.

$\mathrm{OF}+\mathrm{NO}$ tests. The $\mathrm{OF}+\mathrm{NO}$ tests were performed under light conditions of $7 \mathrm{~lx}$. For the OF test, mice were placed in a white open field arena $(50 \times 50 \mathrm{~cm})$ and were allowed to explore the arena for $10 \mathrm{~min}$. For the NO test, experimental animals were then provided an empty water bottle as a novel object into the center of the arena, and were monitored for an additional time of $5 \mathrm{~min}$. Via a video tracking system (Ethovision 11.0 XT, Noldus, Information Technology) the times an animal spend at the different zones of the arena (wall, intermediate, center) and the total distance travelled was calculated.

mCRS paradigm. The mCRS paradigm includes daily restraint of mice in $50 \mathrm{ml}$ falcon tubes equipped with air holes under changing conditions. The order was as follows: (1) $2 \mathrm{~h}$ restraint (9 to $11 \mathrm{am}$ ) at $89 \mathrm{~lx}$ light; (2) $3 \mathrm{~h}$ restraint (10 am to $1 \mathrm{pm})$ in darkness; (3) $1 \mathrm{~h}$ restraint (3 to $4 \mathrm{pm})$ at $89 \mathrm{~lx}$ light and loud music (65-70 dB); (4) $2 \mathrm{~h}$ restraint (9 to $11 \mathrm{am}$ ) at $144 \mathrm{~lx}$ light; (5) $1 \mathrm{~h}$ restraint (4 to $5 \mathrm{pm}$ ) at $89 \mathrm{~lx}$ light and slight jostling on a shaker; (6) $2 \mathrm{~h}$ restraint (10 am to $12 \mathrm{pm}$ ) under stroboscope light; (7) $40 \mathrm{~min}$ restraint (5 to 5:40 pm) at 89 lx light in the presence of 2,3,5-Trimethyl-3-thiazoline (TMT) odor; 8 ) $2 \mathrm{~h}$ restraint (9 to $11 \mathrm{am}$ ) in darkness; 9) $2 \mathrm{~h}$ restraint ( 9 to $11 \mathrm{am}$ ) at $144 \mathrm{~lx}$ light and loud music (65-70 dB); (10) $3 \mathrm{~h}$ restraint (10 am to $1 \mathrm{pm}$ ) at $89 \mathrm{~lx}$ light; (11) $3 \mathrm{~h}$ restraint (1 to $3 \mathrm{pm}$ ) at $144 \mathrm{~lx}$ light; (12) $2 \mathrm{~h}$ restraint ( 9 to $11 \mathrm{am}$ ) at stroboscope light; (13) 30 min restraint (10 to 10:30 am) at 89 lx light in the presence of TMT; (14) $1 \mathrm{~h}$ restraint (4 to $5 \mathrm{pm}$ ) at $89 \mathrm{~lx}$ light and slight jostling 
on a shaker; (15) $3 \mathrm{~h} \mathrm{(10} \mathrm{am} \mathrm{to} 1 \mathrm{pm})$ at $89 \mathrm{~lx}$ light; (16) $2 \mathrm{~h} \mathrm{(2} \mathrm{to} 4 \mathrm{pm})$ at $144 \mathrm{~lx}$ light; (17) $2 \mathrm{~h}$ restraint (9 to $11 \mathrm{am})$ at $89 \mathrm{~lx}$ light and loud music $(65-70 \mathrm{~dB})$; (18) $1 \mathrm{~h}$ restraint (3 to $4 \mathrm{pm})$ under stroboscope light; (19) 40 min restraint (1 to $1: 40 \mathrm{pm}$ ) at $144 \mathrm{~lx}$ light in the TMT odor; (20) $1 \mathrm{~h}$ restraint (10 to $11 \mathrm{am}$ ) at $89 \mathrm{~lx}$ light and slight jostling on a shaker; (21) $2 \mathrm{~h}$ restraint (9 to $11 \mathrm{am}$ ) at $89 \mathrm{~lx}$ light. After 21 days, 30 min of restraint stress at $89 \mathrm{~lx}$ light was continued in the afternoon between the behavioral tests to avoid recovery of the animals from the stressor.

SIT. The SIT ${ }^{94}$ consisting of two consecutive sessions of 2.5 min was performed in a white open field arena $(42 \times 42 \mathrm{~cm})$ at red light conditions. During the first session, animals were allowed to explore the arena including an empty wire mesh. During the second session, a CD1 mouse separated with a wire mesh was introduced into the arena as a social target. With a video tracking system (Ethovision 11.0 XT, Noldus, Information Technology) the time an experimental animal spent in the interaction zone $(24 \times 14 \mathrm{~cm})$ and in the corner zones (each $9 \times 9 \mathrm{~cm}$ ) was measured. Four wild-type animals had to be removed from the analysis due to technical reasons such as an early stopping of the video recording. The social avoidance score was calculated with the following parameters as described in ${ }^{95}$ : (1) the time spend in the interaction zone in the presence of the social target, (2) the time spend in the corner zones in the presence of the social target, (3) the social interaction ratio (time spent in the interaction zone in the presence of the social target/time spent in the interaction zone in the absence of the social target), (4) the corner zone ratio (time spent in the corner zones in the presence of the social target/ time spent in the corner zones in the absence of the social target).

FST. The FST ${ }^{96}$ was performed for 6 min under light conditions of $90 \mathrm{~lx}$. Mice were placed in $5 \mathrm{~L}$ beakers with $3.5 \mathrm{~L}$ of tap water at a temperature of $23-25^{\circ} \mathrm{C}$. Immobility time (the absence of any movement except to keep the animal head above the water) during the last $4 \mathrm{~min}$ of the session was scored manually.

SaPT. For the SaPT, animals were transferred into new home cages with two drinking bottles containing drinking water to habituate them to the new set-up. The next day, one of the bottles was replaced with a $0.05 \%$ saccharin (Sigma, \#S1002) solution and the weight of the water bottles was measured every day for three subsequent days. The bottles were interchanged after each weight measurement to avoid the development of side preference of the animals. Total saccharin consumption was calculated as a percentage of amount of saccharin solution consumed divided by the total volume consumed.

Behavioral z-score index. In order to have an integrated measure of behavioral phenotypes, we performed an integrated behavioral z-score composed of the integrated computation of respective z-normalization across behavioral tests. This approach allows standardizing observations obtained in different experiments, enhancing the reliability of the behavioral phenotyping and increasing analytical possibilities ${ }^{97}$. Specifically, our z-score was calculated by averaging the $\mathrm{z}$-normalized raw data [(x-mean of CTR group)/STD of CTR group] obtained per mouse in the SIT (social avoidance), FST (time of immobility), and SaPT (\% preference) tests. The z-score was determined for mice for which the behavioral parameters could be collected for all tests (i.e., 11 CTR, 16 mCRS mice). In agreement with previous studies ${ }^{42}$, we subdivided chronically stressed mice into low-susceptible (LS) and high-susceptible (HS) clusters according to their z-score. Thus, mCRS mice which fell into the standard deviation from the mean of the control group were designated as LS, as they exhibit comparable behavior to unstressed CTRs, and animals that fell outside were designated as HS.

CORT levels. Trunk blood was collected (sufficient amounts were obtained for 12 CTR and 15 mCRS mice) in heparin-coated capillary tubes (Sarsted, Switzerland) and was centrifuged at $9400 \times g$ at $4{ }^{\circ} \mathrm{C}$ for 4 min to obtain plasma. Basal plasma CORT levels were diluted 20 times and then were measured with an ELISA (Enzo Life Sciences, ADI-901-097 for corticosterone) according to the manufacturer's protocol. Concentration values of CORT were calculated using a 4-parameter logistic fit.

Adrenal glands weight. Adrenal glands were dissected (due to loss during tissue collection, not available for one CTR mouse) and measured to determine adrenal gland weights normalized with body weights of each tested animal [weight adrenal gland in g/body weight in $\mathrm{g}{ }^{\star}$ 100].

Mitochondrial respiratory capacity. PFC and NAc from one hemisphere per animal, counterbalanced across samples $[n=16 \mathrm{CTR}, \mathrm{n}=15 \mathrm{mCRS}$ (due to loss during tissue collection, not available for one stressed animal) for PFC and $n=16$ /group for NAc], was used to measure mitochondrial respiration using the Oroboros Oxygraph $2 \mathrm{~K}$ (Oroboros Instruments, Innsbruck, Austria), as previously described ${ }^{81}$. Briefly, tissue was weight and homogenized in ice-cold respirometry medium $(0.5 \mathrm{mM}$ EGTA, $3 \mathrm{mM} \mathrm{MgCl} 2,60 \mathrm{mM}$ potassium lactobionate, $20 \mathrm{mM}$ taurine, $10 \mathrm{mM} \mathrm{KH} 2 \mathrm{PO} 4,20 \mathrm{mM}$ HEPES, $110 \mathrm{mM}$ sucrose and $0.1 \%(\mathrm{w} / \mathrm{v}) \mathrm{BSA}, \mathrm{pH}=7.1$ ) with an eppendorf pestle. $2 \mathrm{mg}$ of tissue was used to run a multisubstrate protocol at $37^{\circ} \mathrm{C}$ to sequentially assess the various components of mitochondrial respiratory capacity through oxidative phosphorylation (OXPHOS): respiration due to complex I activity (Complex I) was measured by adding $5 \mathrm{mM}$ ADP to a mixture of $2 \mathrm{mM}$ malate, $10 \mathrm{mM}$ pyruvate and $20 \mathrm{mM}$ glutamate, followed by the addition of $10 \mathrm{mM}$ succinate to subsequently stimulate complex II (Complex I + II). Respiration then was uncoupled to examine maximal capacitiy of the electron transport system (ETS) using the protonophore, carbonylcyanide 4 (trifluoromethoxy) phenylhydrazone (FCCP; successive titrations of $0.2 \mu \mathrm{M}$ until maximal respiration rates were reached). The consumption in the uncoupled state due to complex II activity was determined by inhibiting complex I with the addition of $0.1 \mu \mathrm{M}$ 
rotenone (ETS CII). In the last step, electron transport though complex III was inhibited to obtain the level of residual oxygen consumption (ROX) due to oxidating side reactions outside of mitochondrial respiration by adding $2 \mu \mathrm{M}$ antimycin. The $\mathrm{O}_{2}$ flux obtained in each step of the protocol was normalized by the wet weight of the tissue sample used for the analysis and corrected for ROX.

${ }^{1} \mathrm{H}$-magnetic resonance spectroscopy $\left({ }^{1} \mathrm{H}-\mathrm{MRS}\right)$. Another batch of mice ( $\mathrm{n}=8$ /group) was devoted to in vivo ${ }^{1} \mathrm{H}$-MRS measurements of brain metabolites, following procedures previously described in ${ }^{18,54}$. Briefly, all experiments were performed on a horizontal $14.1 \mathrm{~T} / 26 \mathrm{~cm}$ bore animal MR scanner (Magnex Scientific, Abingdon, UK) using a homemade quadrature ${ }^{1} \mathrm{H}$-coil. The volume of interest (VOI) for spectroscopy was placed in the medial PFC (voxel size: $1.4 \times 1.7 \times 1.2 \mathrm{~mm}^{3}$ ) or in the bilateral NAc (voxel size: $4.1 \times 1.4 \times 1 \mathrm{~mm}^{3}$ ) after acquisition of a set of anatomical $\mathrm{T}_{2}$-weighted images for localization. Field homogeneity was adjusted using FAST(EST)MAP to reach a typical water linewidth of $15 \mathrm{~Hz}$ in medial PFC and $20 \mathrm{~Hz}$ in NAc ${ }^{98}$. Spectra were acquired with 20 blocks of 16 averages for medial PFC and 25 blocks of 16 averages for the NAc, leading to a scan duration of around 20 and 25 min respectively. After post-processing of the spectra, metabolite concentrations as well as the Cramér-Rao lower bounds (CRLB) were determined with LCModel using water as internal reference ${ }^{99}$.

RNA isolation, cDNA synthesis, and quantitative RT-PCR. PFC and NAc from one hemisphere per animal, counterbalanced across samples, were snap frozen in liquid nitrogen until use for RNA extraction ( $n=16$ /group). To this end, we used RNAqueous-Micro Total RNA Isolation Kit (ThermoFisher Scientific; \#AM1931) and cDNA synthesis was done with the qScript cDNA SuperMix (QuantaBio; \#733-1176). Quantitative RT-PCR (qRT-PCR) was performed using the SYBR Green PCR Master Mix (Applied Biosystems, Life Technologies, USA) at an ABI Prism 7900 Sequence Detection system (Applied Biosystems, Singapore) with eukaryotic elongation factor $1(E f l a)$ as a reference gene. Primer (Microsynth AG) sequences were as follows: Ef1a fw: tccacttggtcgctttgct, rv: cttcttgtccacagctttgatga; ND-1: fw: ggatgagcctcaaactccaa, rv: ggtcaggctggcagaagtaa; ND-3: fw: gcattctgactcccccaaat, rv: tgaattgctcatggtagtgga; ATP-6: fw: ccttccacaaggaactccaa, rv: ggtagctgttggtgggctaa. Gapdh: fw: tcaccaccatggagaaggc, rv: gctaagcagttggtggtgca.

Bulk RNA-sequencing. The extracted RNA was used for performing the transcriptomic analysis from PFC and NAc of mCRS and unstressed CTR mice [ $n=15 /$ group for PFC (two samples had to be removed from the analysis due to poor sequencing quality); $\mathrm{n}=16$ /group for NAc] using bulk RNA (BRB)-sequencing ${ }^{100}$. Briefly, each RNA sample was reverse transcribed in a 96-well plate using SuperScript II Reverse Transcriptase (Lifetech 18064014) with individual barcoded oligo-dT primers (Microsynth). Next, all the samples were pooled together, purified using the DNA Clean and Concentrator kit (Zymo Research \#D4014), and treated with exonuclease I (NEB or New England BioLabs \#M0293S). Double-stranded cDNA was generated by the second stand synthesis via the nick translation method. For that, a mix containing $2 \mu \mathrm{L}$ of RNAse H (NEB, \#M0297S), $1 \mu \mathrm{L}$ of Escherichia coli DNA ligase (NEB, \#M0205 L), $5 \mu \mathrm{L}$ of E. coli DNA Polymerase (NEB, \#M0209L), $1 \mu \mathrm{L}$ of dNTP (0 $0.2 \mathrm{mM}), 10 \mu \mathrm{L}$ of $5 \times$ Second Stand Buffer (100 mM Tris-HCl (pH 6.9, AppliChem, \#A3452); $25 \mathrm{mM} \mathrm{MgCl} 2$ (Sigma, \#M2670); 450 mM KCl (AppliChem, \#A2939); 0.8 mM $\beta$-NAD Sigma, N1511); 60 mM (NH4)2SO4 (Fisher Scientific Acros, \#AC20587); and $11 \mu \mathrm{L}$ of water was added to $20 \mu \mathrm{L}$ of ExoI-treated first-strand reaction on ice. The reaction was incubated at $16^{\circ} \mathrm{C}$ for $2.5 \mathrm{~h}$. Full-length double-stranded cDNA was purified with $30 \mu \mathrm{L}$ $(0.6 \times)$ of AMPure XP magnetic beads (Beckman Coulter, \#A63881) and eluted in $20 \mu \mathrm{L}$ of water. The Illumina compatible libraries were prepared by tagmentation of $5 \mathrm{ng}$ of full-length double-stranded cDNA with $1 \mu \mathrm{L}$ $(11 \mu \mathrm{M})$ of in-house produced Tn5 enzyme. The final library was amplified 15 cycles and the fragments ranging 200-1000 bp were size-selected using AMPure beads (Beckman Coulter, \#A63881) (first round $0.5 \times$ beads, second $0.7 \times$ ). The libraries were profiled with High Sensitivity NGS Fragment Analysis Kit (Advanced Analytical, \#DNF-474) and measured with Qubit dsDNA HS Assay Kit (Invitrogen, \#Q32851) prior to pooling and sequencing using the Illumina NextSeq 500 platform using a custom primer and the High Output v2 kit (75 cycles) (Illumina, \#FC-404-2005). The library loading concentration was $2.2 \mathrm{pM}$ and sequencing configuration as following: R1 6c/index $8 \mathrm{c} / \mathrm{R} 270 \mathrm{c}$.

Transcriptomic analysis. To assess whether changes in mitochondrial gene expression are a common response to chronic stress, we made use of a publicly available transcriptome data set [GEO accession number: GSE102556; ${ }^{20}$. To this end, we reanalyzed the raw reads of the mouse subseries of this study with a focus on mitochondrial associated gene expression by assessing differential gene expression in PFC ( $n=19 /$ group) and NAc ( $n=20$ /group) between chronic variable stress and unstressed mice. To determine whether MDD subjects exhibit similar changes in mitochondrial gene signatures, we made use of a similar analysis pipeline and applied it on the subseries for MDD subjects of the aforementioned data set [GEO accession number: GSE102556; ${ }^{20}$ ]. We assessed differential gene expression between MDD [NAc: $n=16,8$ males and 8 females; ventromedial (vm) PFC: $n=16,7$ males and 9 females] and healthy subjects [NAc: $n=17,9$ males and 8 females; vmPFC: $n=15,9$ males and 6 females] with available medication status.

Reads produced in this study or publicly available raw reads were mapped with STAR $2.4 .0 \mathrm{~g}^{101}$ onto GRCm38/ $\mathrm{mm} 10$ and GRCh38/hg38 genome assembly for mouse and human data, respectively. Uniquely mapped reads were counted for each gene locus using htseq $0.6 .1^{102}$. We normalized count data by size factor and applied a variance stabilizing transformation for visualization purposes as suggested by Anders et al ${ }^{103}$. We utilized generalized linear model to assess differential gene expression with the help of the DESeq2 package ${ }^{104}$. If not otherwise stated, we contrasted two groups for the mouse data [mCRS vs. CTR (data of this study), and CVS vs. $\mathrm{CTR}^{20}$ ] using a Wald test. To statistically assess differential gene expression between human MDD subjects 
and healthy controls we used a likelihood-ratio to correct differential gene expression for effects from age, RNA quality (RIN), alcohol abuse, medication status and gender as suggested ${ }^{20}$.

Transcription factor activity analysis. To predict transcription factor binding sites of transcription factors in promoters of differentially expressed genes, we employed MotEvo $1.03^{105}$ and scanned \pm 500 bp around the annotated transcription start site (TSS) of a gene. TSS definition were retrieved from ${ }^{106}$ and weight matrices were retrieved from Swiss regulon ${ }^{107}$. To compute transcription factor activity, we applied a penalized regression model described in Balwierz et al. ${ }^{106}$.

Functional and gene set enrichment analysis. For gene set enrichment analysis we employed the camera function of the limma package ${ }^{108}$ on a pre-ranked gene lists defined from the differential gene expression analysis. Gene sets were derived from several sources including AmiGO ${ }^{109}$ for Gene Ontology Biological processes and KEGG ${ }^{110}$. The gene set of mitochondrial associated genes was retrieved from MitoCarta2. $0^{111}$.

Model selection. To assess whether a gene is better explained by the individual behavioral changes of an animal or the absence or presence of mCRS, we applied a model selection approach under the generalized linear model framework of DESeq2. To this end, we defined three models: (1) gene expression explained by z-score, (2) by presence or absence of mCRS or (3) no difference between animals. Each model was solved with generalized linear regression and Bayesian information criterion (BIC) was computed using the deviance of the fit to control for model complexity ${ }^{112}$. We calculated the Schwarz weight to assess model confidence for each model. This analysis was done with animals for which the z-score could be determined and for which transcriptome data were available ( $\mathrm{n}=10$ CTRs, $\mathrm{n}=15 \mathrm{mCRS}$ ).

Statistics. Statistical analyses were performed with Prism 6.0 (Graphpad Software Inc.) and R (R Core Team, 2014). Unless otherwise stated, statistical significance was determined for the comparison of two groups by Student's t-test for data following normal distribution or Mann-Whitney U-test for data following non-normal distribution. For analyses involving more than two groups, a 2-way analysis of variance (ANOVA) followed by Sidak's post hoc test was applied. We log-transformed metabolite levels for spectroscopy and used a linear model to test for the contrast mCRS versus CTR. $p$ values were corrected for multiple testing using the Benjamini-Hochberg method ${ }^{113}$. To test for differences between mCRS and CTR treated mice in respiration experiments, we constructed a linear mixed effects models using the $\mathrm{R}$ package $l m e 4^{114}$. As the measurements for the mitochondrial respiration capacity experiments were performed in blocks of different days, we extracted the $p$ values for the fixed effect treatment and controlled for day of measurement as a random effect. We reported the estimated marginal means of the model. All values represent the mean \pm SEM. Asterisks indicate $p$ value: ${ }^{\star} p<0.05,{ }^{* *} p<0.01,{ }^{* *} p<0.001$.

\section{Data availability}

The generated datasets of this study were made publicly available at the GEO repository (GEO accession number: GSE148629) and can be accessed via the following link: https://www.ncbi.nlm.nih.gov/geo/query/acc. cgi? acc=GSE148629.

Received: 26 June 2020; Accepted: 14 October 2020

Published online: 27 October 2020

\section{References}

1. McEwen, B. S. Physiology and neurobiology of stress and adaptation: Central role of the brain. Physiol. Rev. 87, 873-904 (2007).

2. Sandi, C. Stress, cognitive impairment and cell adhesion molecules. Nat. Rev. Neurosci. 5, 917-930 (2004)

3. de Kloet, E. R., Joëls, M. \& Holsboer, F. Stress and the brain: From adaptation to disease. Nat. Rev. Neurosci. 6, 463-475 (2005).

4. Kessler, R. C. et al. Lifetime prevalence and age-of-onset distributions of DSM-IV disorders in the National Comorbidity Survey Replication. Arch. Gen. Psychiatry 62, 593-602 (2005).

5. Malhi, G. S. \& Mann, J. J. Depression. Lancet (London, England) 392, 2299-2312 (2018).

6. de Kloet, E. R. et al. Stress and depression: A crucial role of the mineralocorticoid receptor. J. Neuroendocrinol. 28 https://doi. org/10.1111/jne.12379 (2016).

7. Troubat, R. et al. Neuroinflammation and depression: A review. Eur. J. Neurosci. https://doi.org/10.1111/ejn.14720 (2020).

8. Ward, J. et al. The genomic basis of mood instability: Identification of 46 loci in 363,705 UK Biobank participants, genetic correlation with psychiatric disorders, and association with gene expression and function. Mol. Psychiatry https://doi.org/10.1038/ s41380-019-0439-8 (2019).

9. Dudek, K. A. et al. Neurobiology of resilience in depression: Immune and vascular insights from human and animal studies. Eur. J. Neurosci. https://doi.org/10.1111/ejn.14547 (2019).

10. Duman, R. S., Sanacora, G. \& Krystal, J. H. Altered connectivity in depression: GABA and glutamate neurotransmitter deficits and reversal by novel treatments. Neuron 102, 75-90 (2019).

11. Muir, J., Lopez, J. \& Bagot, R. C. Wiring the depressed brain: Optogenetic and chemogenetic circuit interrogation in animal models of depression. Neuropsychopharmacology 44, 1013-1026 (2019).

12. Belleau, E. L., Treadway, M. T. \& Pizzagalli, D. A. The impact of stress and major depressive disorder on hippocampal and medial prefrontal cortex morphology. Biol. Psychiatry 85, 443-453 (2019).

13. Russo, S. J. \& Nestler, E. J. The brain reward circuitry in mood disorders. Nat. Rev. Neurosci. 14, 609-625 (2013).

14. Treadway, M. T. \& Pizzagalli, D. A. Imaging the pathophysiology of major depressive disorder-From localist models to circuitbased analysis. Biol. Mood Anxiety Disord. 4, 5 (2014).

15. Kennedy, S. H. et al. Changes in regional brain glucose metabolism measured with positron emission tomography after paroxetine treatment of major depression. Am. J. Psychiatry 158, 899-905 (2001). 
16. Sequeira, A. et al. Patterns of gene expression in the limbic system of suicides with and without major depression. Mol. Psychiatry 12, 640-655 (2007).

17. McKlveen, J. M. et al. Chronic stress increases prefrontal inhibition: A mechanism for stress-induced prefrontal dysfunction. Biol. Psychiatry 80, 754-764 (2016).

18. Larrieu, T. et al. Hierarchical status predicts behavioral vulnerability and nucleus accumbens metabolic profile following chronic social defeat stress. Curr. Biol. 27, 2202-2210.e4 (2017).

19. Chandra, R. et al. Reduced Slc6a15 in nucleus Accumbens D2-neurons underlies stress susceptibility. J. Neurosci. 37, 6527-6538 (2017).

20. Labonté, B. et al. Sex-specific transcriptional signatures in human depression. Nat. Med. 23, 1102-1111 (2017).

21. Seney, M. L. et al. Opposite molecular signatures of depression in men and women. Biol. Psychiatry 84, 18-27 (2018).

22. Nagy, C. et al. Single-nucleus transcriptomics of the prefrontal cortex in major depressive disorder implicates oligodendrocyte precursor cells and excitatory neurons. Nat. Neurosci. https://doi.org/10.1038/s41593-020-0621-y (2020).

23. Krishnan, V. et al. Molecular adaptations underlying susceptibility and resistance to social defeat in brain reward regions. Cell 131, 391-404 (2007).

24. Bagot, R. C. et al. Circuit-wide transcriptional profiling reveals brain region-specific gene networks regulating depression susceptibility. Neuron 90, 969-983 (2016).

25. Hodes, G. E. et al. Sex Differences in nucleus accumbens transcriptome profiles associated with susceptibility versus resilience to subchronic variable stress. J. Neurosci. 35, 16362-16376 (2015).

26. Scarpa, J. R. et al. Shared transcriptional signatures in major depressive disorder and mouse chronic stress models. Biol. Psychiatry https://doi.org/10.1016/j.biopsych.2019.12.029 (2020).

27. Picard, M. et al. Mitochondrial functions modulate neuroendocrine, metabolic, inflammatory, and transcriptional responses to acute psychological stress. Proc. Natl. Acad. Sci. 112, E6614-E6623 (2015).

28. Picard, M., McEwen, B. S., Epel, E. S. \& Sandi, C. An energetic view of stress: Focus on mitochondria. Front. Neuroendocrinol. 49, 72-85 (2018).

29. Filiou, M. D. \& Sandi, C. Anxiety and brain mitochondria: A bidirectional crosstalk. Trends Neurosci. 42, 573-588 (2019).

30. Morava, E. \& Kozicz, T. Mitochondria and the economy of stress (mal)adaptation. Neurosci. Biobehav. Rev. 37, 668-680 (2013).

31. Petschner, P. et al. Genes linking mitochondrial function, cognitive impairment and depression are associated with endophenotypes serving precision medicine. Neuroscience 370, 207-217 (2018).

32. Kasahara, T. \& Kato, T. What can mitochondrial dna analysis tell us about mood disorders?. Biol. Psychiatry 83, 731-738 (2018).

33. Torrell, H. et al. Mitochondrial DNA (mtDNA) in brain samples from patients with major psychiatric disorders: Gene expression profiles, mtDNA content and presence of the mtDNA common deletion. Am. J. Med. Genet. B. Neuropsychiatr. Genet. 162B, 213-223 (2013).

34. Wang, Q. \& Dwivedi, Y. Transcriptional profiling of mitochondria associated genes in prefrontal cortex of subjects with major depressive disorder. World J. Biol. Psychiatry 18, 592-603 (2017).

35. Misiewicz, Z. et al. Multi-omics analysis identifies mitochondrial pathways associated with anxiety-related behavior. PLoS Genet. 15, e1008358 (2019).

36. Duarte, J. M. N., Lei, H., Mlynárik, V. \& Gruetter, R. The neurochemical profile quantified by in vivo ${ }^{1} \mathrm{H}$ NMR spectroscopy. Neuroimage 61, 342-362 (2012).

37. Münch, C. \& Harper, J. W. Mitochondrial unfolded protein response controls matrix pre-RNA processing and translation. Nature 534, 710-713 (2016).

38. Fiorese, C. J. et al. The transcription factor ATF5 mediates a mammalian mitochondrial UPR. Curr. Biol. 26, 2037-2043 (2016).

39. Quirós, P. M. et al. Multi-omics analysis identifies ATF4 as a key regulator of the mitochondrial stress response in mammals. J. Cell Biol. 216, 2027-2045 (2017).

40. Melber, A. \& Haynes, C. M. UPRmt regulation and output: A stress response mediated by mitochondrial-nuclear communication. Cell Res. 28, 281-295 (2018).

41. Polman, J. A. E. et al. Glucocorticoids modulate the mTOR pathway in the hippocampus: Differential effects depending on stress history. Endocrinology 153, 4317-4327 (2012).

42. Nasca, C., Bigio, B., Zelli, D., Nicoletti, F. \& McEwen, B. S. Mind the gap: Glucocorticoids modulate hippocampal glutamate tone underlying individual differences in stress susceptibility. Mol. Psychiatry 20, 755-763 (2015).

43. Kupferberg, A., Bicks, L. \& Hasler, G. Social functioning in major depressive disorder. Neurosci. Biobehav. Rev. 69, 313-332 (2016).

44. Salamone, J. D., Yohn, S. E., López-Cruz, L., San Miguel, N. \& Correa, M. Activational and effort-related aspects of motivation: Neural mechanisms and implications for psychopathology. Brain 139, 1325-1347 (2016).

45. Grahek, I., Shenhav, A., Musslick, S., Krebs, R. M. \& Koster, E. H. W. Motivation and cognitive control in depression. Neurosci. Biobehav. Rev. 102, 371-381 (2019).

46. Hamilton, P. J. et al. Multi-OMIC analysis of brain and serum from chronically-stressed mice reveals network disruptions in purine metabolism, fatty acid beta-oxidation, and antioxidant activity that are reversed by antidepressant treatment. bioRxiv https://doi.org/10.1101/490748 (2018).

47. Holmes, A. \& Wellman, C. L. Stress-induced prefrontal reorganization and executive dysfunction in rodents. Neurosci. Biobehav. Rev. 33, 773-783 (2009)

48. Shansky, R. M. \& Morrison, J. H. Stress-induced dendritic remodeling in the medial prefrontal cortex: Effects of circuit, hormones and rest. Brain Res. 1293, 108-113 (2009).

49. Duman, C. H. \& Duman, R. S. Spine synapse remodeling in the pathophysiology and treatment of depression. Neurosci. Lett. 601, 20-29 (2015).

50. Thompson, S. M. et al. An excitatory synapse hypothesis of depression. Trends Neurosci. 38, 279-294 (2015).

51. Khibnik, L. A. et al. Stress and cocaine trigger divergent and cell type-specific regulation of synaptic transmission at single spines in nucleus accumbens. Biol. Psychiatry 79, 898-905 (2016).

52. Francis, T. C. et al. Molecular basis of dendritic atrophy and activity in stress susceptibility. Mol. Psychiatry 22, 1512-1519 (2017).

53. Gilabert-Juan, J., Castillo-Gomez, E., Guirado, R., Moltó, M. D. \& Nacher, J. Chronic stress alters inhibitory networks in the medial prefrontal cortex of adult mice. Brain Struct. Funct. 218, 1591-1605 (2013).

54. Cherix, A. et al. Metabolic signature in nucleus accumbens for anti-depressant-like effects of acetyl-L-carnitine. Elife $\mathbf{9}$, e50631 (2020).

55. Lopes, S. et al. Tau deletion prevents stress-induced dendritic atrophy in prefrontal cortex: Role of synaptic mitochondria. Cereb. Cortex https://doi.org/10.1093/cercor/bhw057 (2016).

56. Hunter, R. G. et al. Stress and corticosteroids regulate rat hippocampal mitochondrial DNA gene expression via the glucocorticoid receptor. Proc. Natl. Acad. Sci. USA 113, 9099-9104 (2016).

57. Emmerzaal, T. L. et al. Impaired mitochondrial complex I function as a candidate driver in the biological stress response and a concomitant stress-induced brain metabolic reprogramming in male mice. Transl. Psychiatry 10, 176 (2020).

58. Grigoruță, M., Martínez-Martínez, A., Dagda, R. Y. \& Dagda, R. K. Psychological stress phenocopies brain mitochondrial dysfunction and motor deficits as observed in a parkinsonian rat model. Mol. Neurobiol. 57, 1781-1798 (2020). 
59. Kuffner, K. et al. Major depressive disorder is associated with impaired mitochondrial function in skin fibroblasts. Cells 9,884 (2020).

60. Drevets, W. C., Ongür, D. \& Price, J. L. Reduced glucose metabolism in the subgenual prefrontal cortex in unipolar depression. Mol. Psychiatry 3, 190-191 (1998).

61. Drevets, W. C., Price, J. L. \& Furey, M. L. Brain structural and functional abnormalities in mood disorders: Implications for neurocircuitry models of depression. Brain Struct. Funct. 213, 93-118 (2008).

62. MacDonald, K. et al. Biomarkers for major depressive and bipolar disorders using metabolomics: A systematic review. Am. J. Med. Genet. B. Neuropsychiatr. Genet. 180, 122-137 (2019).

63. van der Kooij, M. A. et al. Chronic social stress-induced hyperglycemia in mice couples individual stress susceptibility to impaired spatial memory. Proc. Natl. Acad. Sci. USA 115, E10187-E10196 (2018).

64. Mergenthaler, P., Lindauer, U., Dienel, G. A. \& Meisel, A. Sugar for the brain: The role of glucose in physiological and pathological brain function. Trends Neurosci. 36, 587-597 (2013).

65. Hall, C. N., Klein-Flugge, M. C., Howarth, C. \& Attwell, D. Oxidative phosphorylation, not glycolysis, powers presynaptic and postsynaptic mechanisms underlying brain information processing. J. Neurosci. 32, 8940-8951 (2012).

66. McEwen, B. S. Allostasis and the epigenetics of brain and body health over the life course. JAMA Psychiatry 74, 551 (2017).

67. Picard, M., Juster, R.-P. \& McEwen, B. S. Mitochondrial allostatic load puts the 'gluc' back in glucocorticoids. Nat. Rev. Endocrinol. 10, 303-310 (2014).

68. Du, J. et al. Dynamic regulation of mitochondrial function by glucocorticoids. Proc. Natl. Acad. Sci. 106, 3543-3548 (2009).

69. Musazzi, L. et al. Acute inescapable stress rapidly increases synaptic energy metabolism in prefrontal cortex and alters working memory performance. Cereb. Cortex 29, 4948-4957 (2019).

70. McEwen, B. S. Protection and damage from acute and chronic stress: Allostasis and allostatic overload and relevance to the pathophysiology of psychiatric disorders. Ann. N. Y. Acad. Sci. 1032, 1-7 (2004).

71. Pacelli, C. et al. Elevated mitochondrial bioenergetics and axonal arborization size are key contributors to the vulnerability of dopamine neurons. Curr. Biol. 25, 2349-2360 (2015).

72. Surmeier, D. J. Calcium, ageing, and neuronal vulnerability in Parkinson's disease. Lancet Neurol. 6, 933-938 (2007).

73. Chinta, S. J. \& Andersen, J. K. Redox imbalance in Parkinson's disease. Biochim. Biophys. Acta Gen. Subj. 1780, 1362-1367 (2008).

74. Licznerski, P. \& Duman, R. S. Remodeling of axo-spinous synapses in the pathophysiology and treatment of depression. Neuroscience 251, 33-50 (2013).

75. McEwen, B. S., Nasca, C. \& Gray, J. D. Stress effects on neuronal structure: Hippocampus, amygdala, and prefrontal cortex. Neuropsychopharmacology 41, 3-23 (2016).

76. Stewart, M. G. et al. Stress suppresses and learning induces plasticity in CA3 of rat hippocampus: A three-dimensional ultrastructural study of thorny excrescences and their postsynaptic densities. Neuroscience 131, 43-54 (2005).

77. Magariños, A. M. \& McEwen, B. S. Stress-induced atrophy of apical dendrites of hippocampal CA3c neurons: Comparison of stressors. Neuroscience 69, 83-88 (1995).

78. Vyas, A., Pillai, A. G. \& Chattarji, S. Recovery after chronic stress fails to reverse amygdaloid neuronal hypertrophy and enhanced anxiety-like behavior. Neuroscience 128, 667-673 (2004).

79. Zhang, J.-Y. et al. Chronic stress remodels synapses in an amygdala circuit-specific manner. Biol. Psychiatry 85, 189-201 (2019).

80. Liston, C. et al. Stress-induced alterations in prefrontal cortical dendritic morphology predict selective impairments in perceptual attentional set-shifting. J. Neurosci. 26, 7870-7874 (2006).

81. Hollis, F. et al. Mitochondrial function in the brain links anxiety with social subordination. Proc. Natl. Acad. Sci. USA 112, 15486-15491 (2015).

82. Timmer, M. \& Sandi, C. A role for glucocorticoids in the long-term establishment of a social hierarchy. Psychoneuroendocrinology 35, 1543-1552 (2010).

83. Weger, M. et al. Increased brain glucocorticoid actions following social defeat in rats facilitates the long-term establishment of social subordination. Physiol. Behav. 186, 31-36 (2018).

84. Larrieu, T. \& Sandi, C. Stress-induced depression: Is social rank a predictive risk factor?. BioEssays 40, e1800012 (2018).

85. Barik, J. et al. Chronic stress triggers social aversion via glucocorticoid receptor in dopaminoceptive neurons. Science 339, 332-335 (2013).

86. Douma, E. H. \& de Kloet, E. R. Stress-induced plasticity and functioning of ventral tegmental dopamine neurons. Neurosci. Biobehav. Rev. 108, 48-77 (2020).

87. Adzic, M. et al. Brain region- and sex-specific modulation of mitochondrial glucocorticoid receptor phosphorylation in fluoxetine treated stressed rats: Effects on energy metabolism. Psychoneuroendocrinology 38, 2914-2924 (2013).

88. Moutsatsou, P. et al. Localization of the glucocorticoid receptor in rat brain mitochondria. Arch. Biochem. Biophys. 386, 69-78 (2001).

89. Psarra, A.-M.G. \& Sekeris, C. E. Glucocorticoids induce mitochondrial gene transcription in HepG2 cells: Role of the mitochondrial glucocorticoid receptor. Biochim. Biophys. Acta 1813, 1814-1821 (2011).

90. Nasca, C. et al. L-acetylcarnitine causes rapid antidepressant effects through the epigenetic induction of mGlu2 receptors. Proc. Natl. Acad. Sci. USA 110, 4804-4809 (2013).

91. Poirier, G. L., Imamura, N., Zanoletti, O. \& Sandi, C. Social deficits induced by peripubertal stress in rats are reversed by resveratrol. J. Psychiatr. Res. 57, 157-164 (2014).

92. Hollis, F., Mitchell, E. S., Canto, C., Wang, D. \& Sandi, C. Medium chain triglyceride diet reduces anxiety-like behaviors and enhances social competitiveness in rats. Neuropharmacology 138, 245-256 (2018).

93. Weger, M. \& Sandi, C. High anxiety trait: A vulnerable phenotype for stress-induced depression. Neurosci. Biobehav. Rev. 87, 27-37 (2018).

94. Golden, S. A., Covington, H. E., Berton, O. \& Russo, S. J. A standardized protocol for repeated social defeat stress in mice. Nat. Protoc. 6, 1183-1191 (2011).

95. Anacker, C. et al. Neuroanatomic differences associated with stress susceptibility and resilience. Biol. Psychiatry 79, 840-849 (2016).

96. Porsolt, R. D., Bertin, A. \& Jalfre, M. Behavioral despair in mice: A primary screening test for antidepressants. Arch. Int. Pharmacodyn. Ther. 229, 327-336 (1977).

97. Guilloux, J.-P., Seney, M., Edgar, N. \& Sibille, E. Integrated behavioral z-scoring increases the sensitivity and reliability of behavioral phenotyping in mice: Relevance to emotionality and sex. J. Neurosci. Methods 197, 21-31 (2011).

98. Gruetter, R. \& Tkác, I. Field mapping without reference scan using asymmetric echo-planar techniques. Magn. Reson. Med. 43, 319-323 (2000).

99. Provencher, S. W. Automatic quantitation of localized in vivo ${ }^{1} \mathrm{H}$ spectra with LCModel. NMR Biomed. 14, 260-264 (2001).

100. Alpern, D. et al. BRB-seq: Ultra-affordable high-throughput transcriptomics enabled by bulk RNA barcoding and sequencing. Genome Biol. 20, 71 (2019).

101. Dobin, A. et al. STAR: Ultrafast universal RNA-seq aligner. Bioinformatics 29, 15-21 (2013).

102. Anders, S., Pyl, P. T. \& Huber, W. HTSeq-A Python framework to work with high-throughput sequencing data. Bioinformatics 31, 166-169 (2015).

103. Anders, S. \& Huber, W. Differential expression analysis for sequence count data. Genome Biol. 11, R106 (2010). 
104. Love, M. I., Huber, W. \& Anders, S. Moderated estimation of fold change and dispersion for RNA-seq data with DESeq2. Genome Biol. 15, 550 (2014).

105. Arnold, P., Erb, I., Pachkov, M., Molina, N. \& van Nimwegen, E. MotEvo: Integrated Bayesian probabilistic methods for inferring regulatory sites and motifs on multiple alignments of DNA sequences. Bioinformatics 28, 487-494 (2012).

106. Balwierz, P. J. et al. Methods for analyzing deep sequencing expression data: Constructing the human and mouse promoterome with deepCAGE data. Genome Biol. 10, R79 (2009).

107. Pachkov, M., Balwierz, P. J., Arnold, P., Ozonov, E. \& van Nimwegen, E. SwissRegulon, a database of genome-wide annotations of regulatory sites: Recent updates. Nucleic Acids Res. 41, D214-D220 (2013).

108. Ritchie, M. E. et al. Limma powers differential expression analyses for RNA-sequencing and microarray studies. Nucleic Acids Res. 43, e47 (2015).

109. Carbon, S. et al. AmiGO: Online access to ontology and annotation data. Bioinformatics 25, 288-289 (2009).

110. Kanehisa, M. \& Goto, S. KEGG: Kyoto encyclopedia of genes and genomes. Nucleic Acids Res. 28, 27-30 (2000).

111. Calvo, S. E., Clauser, K. R. \& Mootha, V. K. MitoCarta2.0: An updated inventory of mammalian mitochondrial proteins. Nucleic Acids Res. 44, D1251-D1257 (2016).

112. Kass, R. E. \& Raftery, A. E. Bayes factors. J. Am. Stat. Assoc. 90, 773-795 (1995).

113. Benjamini, Y. \& Hochberg, Y. Controlling the false discovery rate: A practical and powerful approach to multiple testing. J. R. Stat. Soc. Ser. B 57, 289-300 (1995).

114. Bates, D., Mächler, M., Bolker, B. \& Walker, S. Fitting linear mixed-effects models using lme4. J. Stat. Softw. 67, 1-48 (2015).

\section{Acknowledgements}

We would like to thank Dr Benjamin D. Weger for scientific discussions and critically reading the manuscript, and Dr Laia Morato Fornaguera and Dr Thomas Larrieu for scientific discussions. We acknowledge Olivia Zanoletti and Isabelle Guillot de Suduiraut for excellent technical assistance. This project has been supported by grants from the Swiss National Science Foundation [176206; NCCR Synapsy: 51NF40-158776 and -185897] and intramural funding from the EPFL (to CS). MW was awarded with a Marie Curie Intra-European Fellowships for Career Development (EU Project 748051, MitoDyaD).

\section{Author contributions}

M.W. and C.S. designed the research. M.W., D.A., A.C., S.G., J.G., and J.M.R. performed the research. M.W., D.A., and A.C. analyzed the data. M.W., B.D., R.G., and C.S. provided funding and/or supervision. M.W. wrote the first draft and C.S. and E.R.K. contributed to the data interpretation and revision of the manuscript. All authors reviewed and approved the manuscript.

\section{Competing interests}

The authors declare no competing interests.

\section{Additional information}

Supplementary information is available for this paper at https://doi.org/10.1038/s41598-020-75326-9.

Correspondence and requests for materials should be addressed to C.S.

Reprints and permissions information is available at www.nature.com/reprints.

Publisher's note Springer Nature remains neutral with regard to jurisdictional claims in published maps and institutional affiliations.

(c) (1) Open Access This article is licensed under a Creative Commons Attribution 4.0 International cc) License, which permits use, sharing, adaptation, distribution and reproduction in any medium or format, as long as you give appropriate credit to the original author(s) and the source, provide a link to the Creative Commons licence, and indicate if changes were made. The images or other third party material in this article are included in the article's Creative Commons licence, unless indicated otherwise in a credit line to the material. If material is not included in the article's Creative Commons licence and your intended use is not permitted by statutory regulation or exceeds the permitted use, you will need to obtain permission directly from the copyright holder. To view a copy of this licence, visit http://creativecommons.org/licenses/by/4.0/.

(C) The Author(s) 2020 